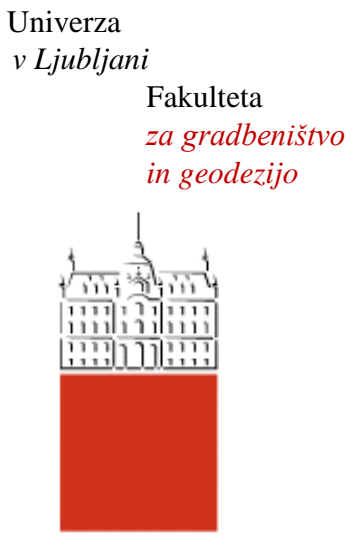

Jamova cesta 2

1000 Ljubljana, Slovenija

http://www3.fgg.uni-lj.si/

\section{DRUGG - Digitalni repozitorij UL FGG http://drugg.fgg.uni-lj.si/}

Ta članek je avtorjeva zadnja recenzirana različica, kot je bila sprejeta po opravljeni recenziji.

Prosimo, da se pri navajanju sklicujte na bibliografske podatke, kot je navedeno:
University
of Ljubljana

Faculty of

Civil and Geodetic

Engineering

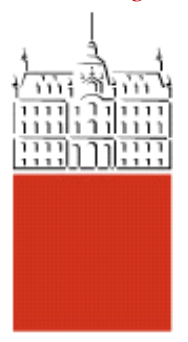

Jamova cesta 2

SI - 1000 Ljubljana, Slovenia

http://www3.fgg.uni-lj.si/en/

DRUGG - The Digital Repository http://drugg.fgg.uni-lj.si/

This version of the article is author's manuscript as accepted for publishing after the review process.

When citing, please refer to the publisher's bibliographic information as follows:

Zupan, D., Saje, M. 2003. Finite-element formulation of geometrically exact threedimensional beam theories based on interpolation of strain measures. Computer Methods in Applied Mechanics and Engineering 192: 5209-5248. DOI: 10.1016/j.cma.2003.07.008. 


\title{
Finite-element formulation of geometrically exact three-dimensional beam theories based on interpolation of strain measures
}

\author{
D. Zupan and M. Saje \\ University of Ljubljana, Faculty of Civil and Geodetic Engineering, Jamova 2, \\ SI-1115 Ljubljana, Slovenia
}

\begin{abstract}
This paper presents a new finite element formulation of the 'geometrically exact finite-strain beam theory'. The governing equations of the beam element are derived in which the strain vectors are the only unknown functions. The consistency condition that the equilibrium and the constitutive internal force and moment vectors are equal, is enforced to be satisfied at chosen points. The solution is found by a collocation algorithm. The linearity of the strain space not only simplifies the application of Newton's method on the non-linear configuration space, but also leads to the strain-objectivity of the proposed method. The accuracy and the efficiency of the derived numerical algorithm are demonstrated by several examples.
\end{abstract}

Key words: non-linear beam theory, finite-element method, three-dimensional rotation, rotational invariant, strain measure

\section{Introduction}

Engineering structures are often modelled by beam models. A number of beam models can be found in scientific literature. In the present paper, we consider the model, derived from the resultant forms of differential equilibrium equations. The associated non-linear strain-displacement equations relate three displacement components and six strain measures (longitudinal and shear strains, and rotational strains) of the axis of a beam. These strain measures are derived in such a way that the relationships between the displacements, the strains, and the stress resultants are consistent with the virtual work principle at the deformed state for any magnitude of displacements, rotations, and strains. The model is often called the 'geometrically exact finite-strain beam theory' and is usually attributed to Reissner [25] and Simo [28]. Many modern finiteelement developers of the three-dimensional beam theories, e.g. Cardona and 
Géradin [6], Ibrahimbegovic [18], Jelenić and Saje [21], Smolénski [30], based their approach on the geometrically exact beam theory.

Geometry of the three-dimensional beam is described by the line of centroids of cross-sections and by the family of the cross-sections not necessarily normal to the line of centroids at the deformed state; therefore, the configuration space of the beam consists of (i) the linear space of position vector of the line of centroids, and (ii) the non-linear space of rotations of cross-sections. Because the spatial rotations are elements of a multiplicative $\mathcal{S O}(3)$ group, the configuration space of the beam is a non-linear manifold. That is what makes the problem of three-dimensional beams so demanding. The way the rotations are parametrized in the theory is crucial; a particular selection of the parametrization has a direct influence on the algorithm and on the form of the tangent stiffness matrix. In the present work, we employ the rotational vector for the parametrization of rotations.

The essential part of any finite-element formulation is the choice of the primary variables. Earlier finite-element beam formulations use both, displacements and rotations, as the interpolated degrees of freedom (e.g., Cardona and Géradin [6], Crisfield [8], Crivelli and Felippa [10], Ibrahimbegovic [18], Simo and Vu-Quoc [29]), or solely rotations (Jelenić and Saje [21]). These approaches interpolate the rotations or/and their increments in a standard manner, neglecting the fact that the rotations are physically non-additive quantities. As reported by Crisfield and Jelenić [9], such an approach leads to non-objective discrete strain measures, when they are calculated from the interpolated rotations and displacements. Crisfield and Jelenić [9] suggest a strain-objective formulation, requiring a construction of special interpolating functions, which is a complicated task if higher-order finite elements are to be derived. Ibrahimbegovic and Taylor [20] claim that the frame-invariance can be preserved only by proper implementation of the geometrically exact model, yet their definition of the frame-invariance somewhat differs from Crisfield and Jelenić's.

Although the rotations are essential to the overall deformation of the structure, it is their derivative with respect to axis coordinate and not the rotations themselves that effects the deformation energy. This suggests that strain measures and not rotations are natural variables for the description of the deformation energy. Therefore, and in contrast to the above mentioned formulations, we here assume that strain measures are primary interpolated variables. For the strain approximation, the additive-type of interpolation can be used without a loss of the objectivity of the discrete strain measures, i.e., their invariance to rigid-body motions. The displacement and rotational vectors are not interpolated at all. Our formulation experiences no kind of locking, and, compared to other formulations, gives higher accuracy for the given number of external degrees of freedom. It enables more accurate descriptions of strain and stress 
distributions within an element, which is of utmost importance in describing the behaviour of plastic material in the regions of localized strain. In order to apply the strain measures as basic variables, we follow the work by Planinc et al. [24] and extend it to three-dimensional beams and frames by proposing a modified principle of virtual work in which only the strain vectors need to be interpolated.

The fundamental problem of such a strain-based formulation is the integration of rotations from the given interpolated rotational strains. In the planar case, the integration can be performed analytically. In three dimensions, the derivative of rotations equals the product of a rotation-dependent transformation matrix and the rotational strain. Such a system of differential equations cannot be integrated in a closed form. That is why, in three-dimensional beam theories, the rotational strain is very rarely chosen as the primary variable. Such formulations were proposed by Choi and Lim [7], Friedman and Kosmatka [14], who used constant interpolation for curvature, and by $\mathrm{Hu}$ et al. [16], who used a linear strain interpolation. Tabarrok et al. [32] assumed an analytically integrable curvature distribution in order to develop a more suitable interpolation for displacements and rotations. Schulz and Filippou [27] interpolated the infinitesimal (incremental) curvatures and the infinitesimal rotations; in such a formulation, the objectivity of the total rotations and the total curvatures may be questioned. In a sharp contrast to the above cited authors, our solution employs the exact relationship between the total rotational strains (here also called curvatures) and the rotations. Due to the complicated form of the kinematical equations, a numerical integration method is used to obtain the total rotations from the given rotational strains. The linearity of the space of basic variables - the strains - simplifies Newton's iteration method when compared to the case where the rotations are basic variables, and leads automatically to the objectivity of the interpolated strains.

In standard finite-element formulations, the stress-resultants, obtained from the equilibrium equations, and those found from the constitutive equations, are not equal. In the present formulation this 'inconsistency of equilibrium at cross-sections' is resolved by enforcing the consistency condition to be satisfied at a set of predefined points (here taken to coincide with the interpolation points) (the 'collocation'). A similar strategy was employed by Vratanar and Saje [33] for the elastic-plastic analysis of plane frames. Further advantage of the present formulation is its ability not to require the differentiation with respect to the arc-length to determine internal forces; hence, the accuracy of internal forces is of the same order as the accuracy of basic variables. 


\section{Geometry and kinematics of the three-dimensional beam}

\subsection{Basic kinematic assumptions}

The geometry of the three-dimensional beam is described by the family of plane cross-sections and by the line of centroids of cross-sections. The geometric shape of the cross-sections is arbitrary and constant along the length of the beam. According to the Bernoulli hypothesis plane cross-sections are assumed to remain planar and preserve their shape and area after the deformation. Thus the geometric model of the beam consists of a set of

(i) a spatial curve representing the line of centroids, and

(ii) a family of spatial planes containing the cross-sections (Figure 1).

Note that the cross-sections are not necessarily normal to the line of centroids at their intersections. This makes it possible to include the effect of shear stresses into the beam analysis.

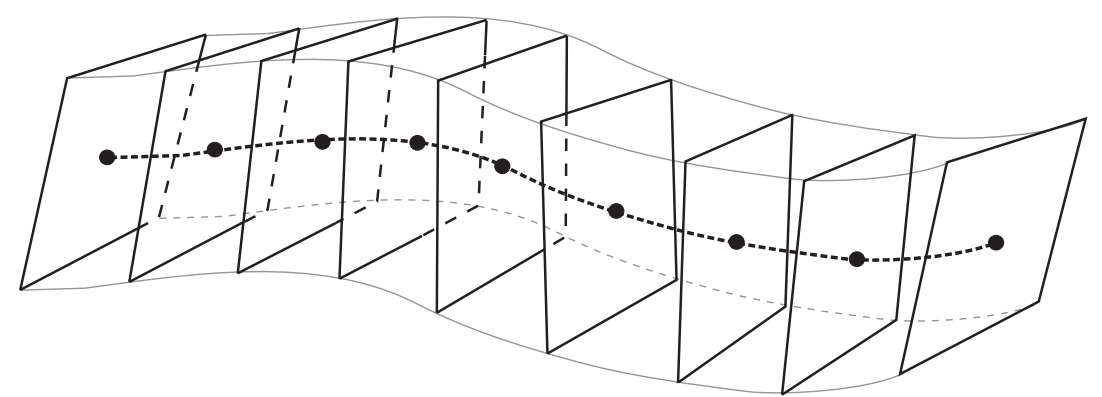

Fig. 1. Abstract model of the three-dimensional beam: a curve and a family of planes.

It is convenient to define

(i) the reference configuration, where the mechanical variables of the beam, including the loading, are known, and

(ii) an arbitrary deformed configuration, where only the loading is prescribed, while the remaining mechanical variables are unknown.

The geometry of the reference configuration (see Figure 2) is described by the family of position vectors $\vec{r}^{0}$ of the line of centroids and by the family of orthonormal base vectors $\left\{\vec{G}_{1}^{0}, \vec{G}_{2}^{0}, \vec{G}_{3}^{0}\right\}$ spanning the planes of cross-sections. Vectors $\vec{G}_{2}^{0}$ and $\vec{G}_{3}^{0}$ are directed along the principal axes of inertia of the 
particular cross-section, and $\vec{G}_{1}^{0}$ is its normal: $\vec{G}_{1}^{0}=\vec{G}_{2}^{0} \times \vec{G}_{3}^{0}$. The reference line of centroids is parametrized by the arc-length, $x$. Vectors $\vec{r}^{0}, \vec{G}_{1}^{0}, \vec{G}_{2}^{0}$, and $\vec{G}_{3}^{0}$ are then expressed as the vector functions of $x$. A deformed configuration is analogously described by four vector functions $\vec{r}(x), \vec{G}_{1}(x), \vec{G}_{2}(x)$, and $\vec{G}_{3}(x)$. Note that the deformed configuration is also expressed with respect to the arc-length parameter of the reference line of centroids. Vectors $\vec{G}_{2}$ and $\vec{G}_{3}$ are directed along the principal axes of inertia of the rotated cross-section, and $\vec{G}_{1}$ is its normal vector, $\vec{G}_{1}=\vec{G}_{2} \times \vec{G}_{3}$. As the basis $\left\{\vec{G}_{1}, \vec{G}_{2}, \vec{G}_{3}\right\}$ is different at each material point, $x$, of the line of centroids, it is called the "material basis'.

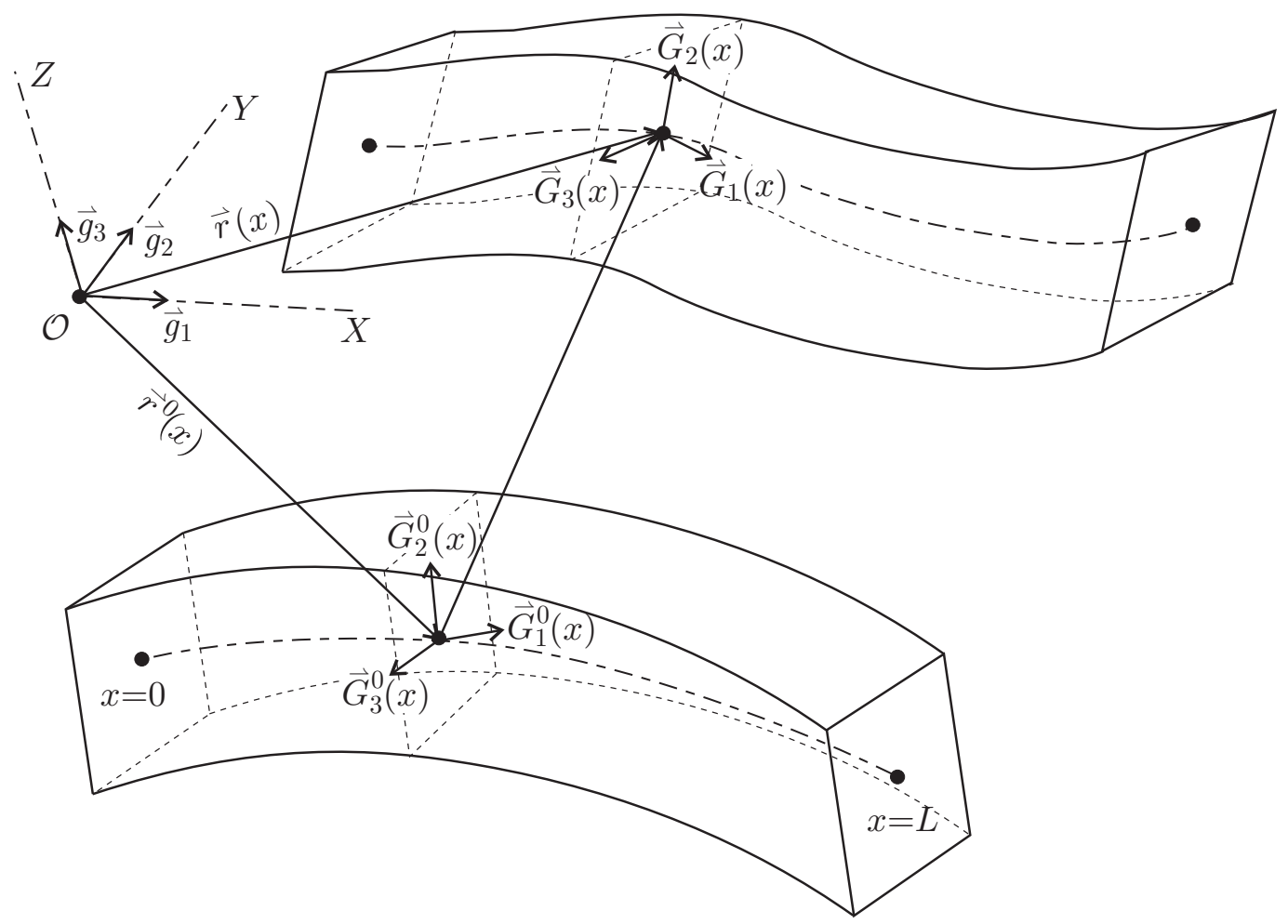

Fig. 2. Parametrization of the three-dimensional beam in the abstract vector space.

We find convenient the introduction of an (arbitrary) reference point $\mathcal{O}$ and a triad of fixed orthonormal base vectors $\left\{\vec{g}_{1}, \vec{g}_{2}, \vec{g}_{3}\right\}$. The triad $\left\{\vec{g}_{1}, \vec{g}_{2}, \vec{g}_{3}\right\}$ is not related to the deformation of the beam and is henceforth referred to as the 'spatial basis', since it spans the physical space of the beam - the Euclidean linear vector space $\mathbb{R}^{3}$. Along with a reference point $\mathcal{O}$, the basis $\left\{\vec{g}_{1}, \vec{g}_{2}, \vec{g}_{3}\right\}$ defines the spatial Cartesian coordinate system $(X, Y, Z)$. With respect to the spatial basis, the rotated position of the cross-sections can 
be described by the rotational operator $\mathcal{R}(x)$, which maps $\left\{\vec{g}_{1}, \vec{g}_{2}, \vec{g}_{3}\right\}$ into $\left\{\vec{G}_{1}(x), \vec{G}_{2}(x), \vec{G}_{3}(x)\right\}:$

$$
\vec{G}_{i}=\mathcal{R} \vec{g}_{i}, \quad i=1,2,3 .
$$

Operator $\mathcal{R}(x)$ is formally a linear operator on the abstract three-dimensional Euclidean space and represents the physical rotation between the two bases. When we express each vector $\vec{G}_{i}$ as the linear combination of base vectors $\left\{\vec{g}_{1}, \vec{g}_{2}, \vec{g}_{3}\right\}$, the components of vector $\vec{G}_{i}$ are just the column components of the rotation matrix:

$$
\vec{G}_{i}=\mathrm{R}_{1 i} \vec{g}_{1}+\mathrm{R}_{2 i} \vec{g}_{2}+\mathrm{R}_{3 i} \vec{g}_{3}, \quad i=1,2,3 .
$$

Thus, the $i$-th column of the rotation matrix $\mathbf{R}=\left[\mathrm{R}_{j i}\right], i, j=1,2,3$, represents the components of the base vector $\vec{G}_{i}$ with respect to the spatial basis. That is why we say that the rotation matrix, $\mathbf{R}$, is associated with the rotational operator, $\mathcal{R}$, with respect to the spatial basis.

An arbitrary vector, $\vec{u}$, can be expressed with respect to either of the two bases

$$
\vec{u}=u_{g 1} \vec{g}_{1}+u_{g 2} \vec{g}_{2}+u_{g 3} \vec{g}_{3}=u_{G 1} \vec{G}_{1}+u_{G 2} \vec{G}_{2}+u_{G 3} \vec{G}_{3}
$$

For the sake of simplicity, the components of the vector, $\left\{u_{g 1}, u_{g 2}, u_{g 3}\right\}$ and $\left\{u_{G 1}, u_{G 2}, u_{G 3}\right\}$, are also represented in the matrix form by one-column matrices

$$
\boldsymbol{u}_{g}=\left[\begin{array}{c}
u_{g 1} \\
u_{g 2} \\
u_{g 3}
\end{array}\right], \quad \boldsymbol{u}_{G}=\left[\begin{array}{c}
u_{G 1} \\
u_{G 2} \\
u_{G 3}
\end{array}\right] .
$$

Both, $\boldsymbol{u}_{g}$ and $\boldsymbol{u}_{G}$, along with the corresponding bases, equivalently represent the vector $\vec{u}$. Inserting (2) into (3) gives the relationship between the two one-column matrices, $\boldsymbol{u}_{g}$ and $\boldsymbol{u}_{G}$,

$$
\boldsymbol{u}_{g}=\mathbf{R} \boldsymbol{u}_{G}
$$

Here, another meaning of the rotation matrix is revealed: not only that it rotates a vector, it also represents the coordinate transformation between the components of a vector with respect to spatial and material bases.

In what follows, vectors will be used in both forms, the abstract and the component (matrix) forms. The abstract vector form will be used in the derivation 
of the beam governing equations. The matrix form will be used in the formulation of the numerical solution.

\subsection{Parametrization of rotations, angular velocity, variation of rotations}

Only some basic aspects and results, needed for further use, are presented here. Fundamentals on rotations in computational mechanics can be found in many papers (e.g., Argyris [1], Atluri and Cazzani [3], Géradin and Rixen $[15])$.

When the linear rotational operator is expressed by the associated matrix, nine scalar components are needed. It can be proved, however, that not all of them are independent. In fact, only three scalar values suffice to describe fully the rotation in the three-dimensional space. There is a number of possibilities for choosing three independent rotational parameters. Here we choose the rotational vector $\vec{\vartheta}$ [1] that lies on the axis of rotation and has its length equal to the angle of rotation. The rotational operator $\mathcal{R}$ is dependent directly on the rotational vector, and the relationship between the two quantities was given by Rodrigues:

$$
\mathcal{R} \vec{u}=\vec{u}+\frac{\sin \vartheta}{\vartheta} \vec{\vartheta} \times \vec{u}+\frac{1-\cos \vartheta}{\vartheta^{2}} \vec{\vartheta} \times(\vec{\vartheta} \times \vec{u}) .
$$

$\vec{u}$ denotes an arbitrary vector and ' $x$ ' the cross vector product; $\vartheta=\|\vec{\vartheta}\|$ is the length of rotational vector $\vec{\vartheta}$ (i.e., the angle of rotation).

If we define a linear operator $\Theta$ so that

$$
\Theta \vec{u}=\vec{\vartheta} \times \vec{u}
$$

the Rodrigues rotational operator can be rewritten in the following form

$$
\mathcal{R}=\mathcal{I}+\frac{\sin \vartheta}{\vartheta} \Theta+\frac{1-\cos \vartheta}{\vartheta^{2}} \Theta^{2}
$$

with $\mathcal{I}$ being an identity operator. Formula (6) will be referred to as the Rodrigues formula. When expressed with respect to an orthonormal basis, the operator $\Theta$ is associated with the skew-symmetric matrix $\boldsymbol{\Theta}$

$$
\boldsymbol{\Theta}=\left[\begin{array}{ccc}
0 & -\vartheta_{3} & \vartheta_{2} \\
\vartheta_{3} & 0 & -\vartheta_{1} \\
-\vartheta_{2} & \vartheta_{1} & 0
\end{array}\right] .
$$


Components $\left\{\vartheta_{1}, \vartheta_{2}, \vartheta_{3}\right\}$ in $(7)$ are the components of vector $\vec{\vartheta}$ with respect to the particular orthonormal basis chosen. From (7) it directly follows that the matrix $\boldsymbol{\Theta}$ is skew-symmetric: $\boldsymbol{\Theta}^{T}=-\boldsymbol{\Theta}$. Once the matrix representation of operator $\Theta$ is known, the matrix form of the rotational operator (6) follows easily

$$
\mathbf{R}=\mathbf{I}+\frac{\sin \vartheta}{\vartheta} \boldsymbol{\Theta}+\frac{1-\cos \vartheta}{\vartheta^{2}} \boldsymbol{\Theta}^{2}
$$

where $\mathbf{I}$ is the identity matrix.

The inverse of the rotational operator (matrix) is easy to find. As $\mathcal{R}$ is a linear operator, there exists such a unique linear operator $\mathcal{R}^{t}$ that

$$
(\mathcal{R} \vec{x}) \cdot \vec{y}=\vec{x} \cdot\left(\mathcal{R}^{t} \vec{y}\right) \quad \text { for all vectors } \vec{x} \text { and } \vec{y} \text {. }
$$

The $\operatorname{dot}(\cdot)$ denotes the scalar product of vectors. $\mathcal{R}^{t}$ is called the transpose of $\mathcal{R}$ and its associated matrix equals the transpose of matrix $\mathbf{R}: \mathcal{R}^{t} \rightarrow \mathbf{R}^{T}$.

The application of definition (9) to operator $\Theta$ gives its transpose

$$
(\Theta \vec{x}) \cdot \vec{y}=(\vec{\vartheta} \times \vec{x}) \cdot \vec{y}=-\vec{x} \cdot(\vec{\vartheta} \times \vec{y})=-\vec{x} \cdot(\Theta \vec{y})=\vec{x} \cdot\left(\Theta^{t} \vec{y}\right) .
$$

We see that the operator $\Theta$ is skew-symmetric: $\Theta^{t}=-\Theta$. From the Rodrigues formula and the skew-symmetry of $\Theta$, it is easy to see that $\mathcal{R}$ is invertible, its inverse being $\mathcal{R}^{t}$

$$
\mathcal{R}^{-1}=\mathcal{R}^{t} \quad \rightarrow \quad \mathcal{R} \mathcal{R}^{t}=\mathcal{R}^{t} \mathcal{R}=\mathcal{I}
$$

Also, by analogy, $\mathbf{R}$ is invertible and its inverse equals $\mathbf{R}^{T}$.

The derivative of material base vectors with respect to $x$ plays an important role in the beam theories. The derivation of (1) with respect to $x$ gives

$$
\vec{G}_{i}^{\prime}=\mathcal{R}^{\prime} \vec{g}_{i}=\mathcal{R}^{\prime} \mathcal{R}^{t} \vec{G}_{i}
$$

In the kinematics of rigid bodies, where the parameter $x$ is replaced by time, operator $\Omega=\mathcal{R}^{\prime} \mathcal{R}^{t}$ is called the angular velocity of the base vector $\vec{G}_{i}$. In the present paper, $\Omega$ describes the rate of change of the material basis with respect to $x$; therefore, the geometrical term 'curvature' would be more adequate. As a consequence of the effect of shear stresses, base vector $\vec{G}_{1}(x)$ differs from the tangent vector $\frac{d \vec{r}}{d x}$ of the axis. Hence, the curvature of the line of centroids and $\Omega$ are different quantities and the term 'pseudo-curvature' is used for $\Omega$ instead. It is easy to see that the operator $\Omega$ is skew-symmetric; consequently there exists such a unique vector $\vec{\omega}$ that

$$
\Omega \vec{u}=\vec{\omega} \times \vec{u} \quad \text { for any } \vec{u} .
$$


In the kinematics of rigid bodies, $\vec{\omega}$ would be called the angular velocity vector. According to the previous discussion, however, we will rather use a different notation, $\vec{\kappa}$, for the axial vector

$$
\Omega \vec{u}=\vec{\kappa} \times \vec{u} \quad \text { for any } \vec{u},
$$

and call it the curvature vector (or sometimes the pseudo-curvature vector).

We can show (see, e.g., [3], [19]) that the curvature vector can be expressed by the derivative of the rotational vector as

$$
\vec{\kappa}=\mathcal{T} \vec{\vartheta}^{\prime}
$$

$\mathcal{T}$ is a linear operator, given as a (non-linear) function of rotational vector $\vec{\vartheta}$ and expressed in an explicit operator form by

$$
\mathcal{T}=\mathcal{I}+\frac{1-\cos \vartheta}{\vartheta^{2}} \Theta+\frac{\vartheta-\sin \vartheta}{\vartheta^{3}} \Theta^{2}
$$

or with its image on an arbitrary vector $\vec{u}$

$$
\mathcal{T} \vec{u}=\vec{u}+\frac{1-\cos \vartheta}{\vartheta^{2}} \vec{\vartheta} \times \vec{u}+\frac{\vartheta-\sin \vartheta}{\vartheta^{3}} \vec{\vartheta} \times(\vec{\vartheta} \times \vec{u}) .
$$

Observe that the operator $\mathcal{T}$, although it operates on the vector space as a linear operator, is a non-linear function of rotational vector $\vec{\vartheta}$. In the planar case, $\mathcal{T}$ equals the identity operator $\mathcal{I}$; this fact considerably simplifies the planar problem.

The variation of a functional will here be made in the sense of the Gâteaux derivative. If the limit

$$
\delta \mathcal{F}(\vec{w})=\lim _{\alpha \rightarrow 0} \frac{\mathcal{F}(\vec{u}+\alpha \vec{w})-\mathcal{F}(\vec{u})}{\alpha}=\left.\frac{d}{d \alpha}\right|_{\alpha=0} \mathcal{F}(\vec{u}+\alpha \vec{w})
$$

exists for arbitrary vectors $\vec{u}$ and $\vec{w}$, it is called the Gâteaux variation of functional $\mathcal{F}$ at $\vec{u}$ in the direction $\vec{w}$. Note that the definition of the Gâteaux variation of a functional assumes that $\mathcal{F}$ is a functional on a linear (additive) vector space. Because the rotational functional $\mathcal{R}$ is expressed in terms of the non-additive rotational vector $\vec{\vartheta}$, the computation of the difference requires a special treatment. If the change of $\vec{\vartheta}$ is denoted by $\alpha \vec{\delta}$, the rotational operator at the perturbed value of its argument is $\mathcal{R}(\alpha \vec{\vartheta}) \mathcal{R}(\vec{\vartheta})$, so that the difference between the original and the perturbed value of the rotational operator is

$$
\Delta \mathcal{R}=\mathcal{R}(\alpha \delta \vec{\vartheta}) \mathcal{R}(\vec{\vartheta})-\mathcal{R}(\vec{\vartheta})
$$


The Gâteaux variation of $\mathcal{R}$ is then evaluated by the definition

$$
\delta \mathcal{R}=\left.\frac{d}{d \alpha}\right|_{\alpha=0} \mathcal{R}(\alpha \dot{\vec{\vartheta}}) \mathcal{R}(\vec{\vartheta}) .
$$

Observe that the vector argument of $\mathcal{R}$, given in the parentheses, is the vector that parametrizes functional $\mathcal{R}$, and not the vector which $\mathcal{R}$ works upon. From (6) it follows that

$$
\mathcal{R}(\alpha \delta \vec{\vartheta})=\mathcal{I}+\frac{\sin (\alpha \delta \vartheta)}{\alpha \delta \vartheta} \alpha \delta \Theta+\frac{1-\cos (\alpha \delta \vartheta)}{(\alpha \delta \vartheta)^{2}}(\alpha \delta \Theta)^{2}
$$

where $\delta \Theta$ is the skew-symmetric operator corresponding to vector $\delta \vec{\vartheta}$ by relation $\delta \Theta \vec{u}=\delta \vec{\vartheta} \times \vec{u}$. Taking the derivative with respect to $\alpha$ and evaluating the result at $\alpha=0$, we obtain

$$
\delta \mathcal{R}=\delta \Theta \mathcal{R}(\Theta)
$$

\section{Strain measures and stress resultants}

We consider a beam subjected to the external distributed force and moment vectors $\vec{n}$ and $\vec{m}$ per unit length of the reference line of centroids. The stressresultant force vector over the cross-section is denoted by $\vec{N}$ and the resulting moment vector by $\vec{M}$. The equilibrium equations of an infinitesimal element of a beam, as illustrated in Figure 3, are given by the following differential equations:

$$
\begin{aligned}
& \vec{n}(x)=-\vec{N}^{\prime}(x), \\
& \vec{m}(x)=-\vec{M}^{\prime}(x)-\vec{r}^{\prime}(x) \times \vec{N}(x) .
\end{aligned}
$$

Following the approach of Reissner [25], we introduce strain vectors $\vec{\gamma}$ and $\vec{\kappa}$ in such a way as to be consistent with the virtual work principle for an arbitrary part of the beam, bounded by the cross-sections at $x=x_{1}$ and $x=x_{2}$ :

$$
\begin{aligned}
\int_{x_{1}}^{x_{2}} \vec{N} \cdot \delta(\vec{\gamma})_{\text {rel }} d x+\int_{x_{1}}^{x_{2}} \vec{M} \cdot \delta(\vec{\kappa})_{\text {rel }} d x=\int_{x_{1}}^{x_{2}} \vec{n} \cdot \delta \vec{r} d x \\
+\int_{x_{1}}^{x_{2}} \vec{m} \cdot \delta \vec{\vartheta} d x+[\vec{N} \cdot \delta \vec{r}+\vec{M} \cdot \delta \vec{\vartheta}]_{x_{1}}^{x_{2}}
\end{aligned}
$$

Note that the virtual work principle is written in an abstract vector form, in which the relative variation of strain vectors is introduced. The relative 


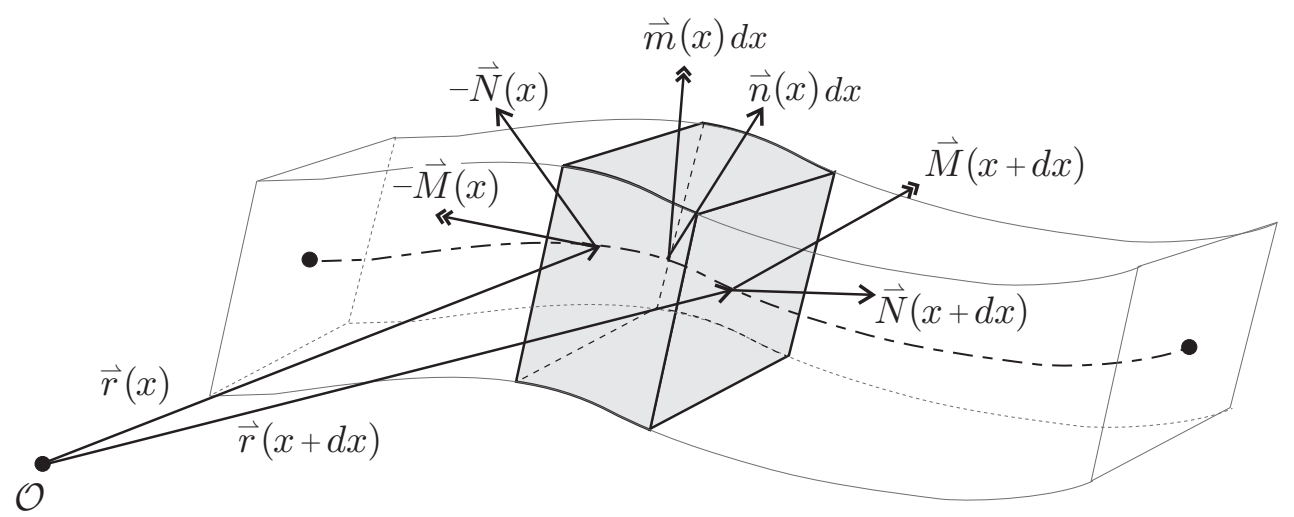

Fig. 3. Equilibrium of an infinitesimal element of a beam.

variation of a vector is a partial variation of a vector that disregards the variation of rotations. It is determined by the following equation

$$
\delta(\vec{u})_{\mathrm{rel}}=\delta \vec{u}-\delta \vec{\vartheta} \times \vec{u} .
$$

This is in accord with the notion of 'objective (or corotated) rates'; see, e.g., Simo [28]. In order to describe the term 'relative' more precisely, let us evaluate the relative variation of a vector, expressed with respect to the material basis. The variation of such a vector consists of the variation of components and the variation of base vectors

$$
\delta \vec{u}=\delta u_{G 1} \vec{G}_{1}+\delta u_{G 2} \vec{G}_{2}+\delta u_{G 3} \vec{G}_{3}+u_{G 1} \delta \vec{G}_{1}+u_{G 2} \delta \vec{G}_{2}+u_{G 3} \delta \vec{G}_{3} .
$$

The variation of the material base vectors $\delta \vec{G}_{i}$ is obtained by the use of the variation of the rotational operator (12) and can be expressed by

$$
\delta \vec{G}_{i}=\delta \vec{\vartheta} \times \vec{G}_{i}
$$

By inserting (17) and (18) into (16) we obtain the formula for the relative variation

$$
\delta(\vec{u})_{\mathrm{rel}}=\delta u_{G 1} \vec{G}_{1}+\delta u_{G 2} \vec{G}_{2}+\delta u_{G 3} \vec{G}_{3} .
$$

Inserting equations (13)-(14) into virtual work equation (15) and applying the partial integration yield the relationships between the variations of kinematic vector variables $(\vec{r}, \vec{\vartheta})$ and the relative variations of strain vectors $(\vec{\gamma}, \vec{\kappa})$

$$
\begin{aligned}
& \delta(\vec{\gamma})_{\text {rel }}=\delta \vec{r}^{\prime}-\delta \vec{\vartheta} \times \vec{r}^{\prime} \\
& \delta(\vec{\kappa})_{\text {rel }}=\delta \vec{\vartheta}^{\prime} .
\end{aligned}
$$

Equations (20) and (21) relate the variations of strains, displacements, and rotations, and indicate that the variations of the quantities mentioned above 
are not all independent. By integrating equations (20) and (21) and following the approach similar to that of Reissner [25], we obtain the relation between strain measures $(\vec{\gamma}, \vec{\kappa})$ and displacements and rotations $(\vec{r}, \vec{\vartheta})$

$$
\begin{aligned}
& \vec{\gamma}=\vec{r}^{\prime}+\vec{c} \\
& \vec{\kappa}=\mathcal{T} \vec{\vartheta}^{\prime}+\vec{d} .
\end{aligned}
$$

Vector functions $\vec{c}(x)$ and $\vec{d}(x)$ are variational constants $\left(\delta(\vec{c})_{\mathrm{rel}}=\delta(\vec{d})_{\mathrm{rel}}=\right.$ $\overrightarrow{0})$ to be determined from the known strains and kinematics of the beam at the reference configuration.

The result (22) can be easily proven. As $\delta \vec{r}^{\prime}-\delta \vec{\vartheta} \times \vec{r}^{\prime}$ equals the relative variation of vector $\vec{r}^{\prime}$, equation (20) gives

$$
\delta(\vec{\gamma})_{\mathrm{rel}}=\delta\left(\vec{r}^{\prime}\right)_{\mathrm{rel}},
$$

from which it is apparent that $\vec{\gamma}$ and $\vec{r}^{\prime}$ differ only in the variational constant $\vec{c}$, for which

$$
\delta(\vec{c})_{\mathrm{rel}}=\overrightarrow{0}
$$

must hold. The equation says that if $\vec{c}$ is expressed with respect to the material basis, its components remain the same during the deformation, because their relative variations equal zero. Note, however, that $\vec{c}$ is still an arbitrary function of $x$.

The proof of the solution in (23) is not as obvious. From (21) such a vector is sought that its relative variation should equal $\delta \vec{\vartheta}^{\prime}$. Vector $\vec{\vartheta}^{\prime}$ is associated with a skew-symmetric operator $\Theta^{\prime}$, which is related to skew-symmetric operator $\mathcal{R}^{\prime} \mathcal{R}^{t}=\Omega$. Both, $\Omega$ and $\Theta^{\prime}$, each in its own way, represent the rate of change of the material basis with respect to $x$. Let us first find the variation of $\Omega$ :

$$
\begin{aligned}
\delta \Omega & =\delta \mathcal{R}^{\prime} \mathcal{R}^{t}+\mathcal{R}^{\prime} \delta \mathcal{R}^{t}=(\delta \Theta \mathcal{R})^{\prime} \mathcal{R}^{t}+\mathcal{R}^{\prime}(\delta \Theta \mathcal{R})^{t} \\
& =\delta \Theta^{\prime} \mathcal{R} \mathcal{R}^{t}+\delta \Theta \mathcal{R}^{\prime} \mathcal{R}^{t}+\mathcal{R}^{\prime} \mathcal{R}^{t} \delta \Theta^{t} .
\end{aligned}
$$

From $\mathcal{R} \mathcal{R}^{t}=\mathcal{I}, \delta \Theta^{t}=-\delta \Theta$ and from the above equation it follows

$$
\delta \Omega=\delta \Theta^{\prime}+\delta \Theta \Omega-\Omega \delta \Theta
$$

If we apply $\delta \Omega$ on an arbitrary vector $\vec{u}$, we get

$$
\begin{aligned}
\delta \Omega \vec{u} & =\delta \Theta^{\prime} \vec{u}+(\delta \Theta \Omega-\Omega \delta \Theta) \vec{u} \\
& =\delta \vec{\vartheta}^{\prime} \times \vec{u}+(\delta \vec{\vartheta} \times \vec{\omega}) \times \vec{u} .
\end{aligned}
$$


On the other hand, we know that $\Omega \vec{u}=\vec{\kappa} \times \vec{u}$, holds true thus

$$
\delta \Omega \vec{u}=\delta \vec{\kappa} \times \vec{u}
$$

Combining (26) and (27) gives

$$
\delta \vec{\kappa}=\delta \vec{\vartheta}^{\prime}+\delta \vec{\vartheta} \times \vec{\omega} \rightarrow \delta \vec{\kappa}-\delta \vec{\vartheta} \times \vec{\kappa}=\delta \vec{\vartheta}^{\prime}
$$

and

$$
\delta(\vec{\kappa})_{\mathrm{rel}}=\delta \vec{\vartheta}^{\prime}
$$

which concludes the proof.

We assume that the stress-resultant vectors, $\vec{N}$ and $\vec{M}$, depend directly on strains $\vec{\gamma}$ and $\vec{\kappa}$. Since we do not wish to restrict our analysis to a particular class of materials of the beam, we assume a rather general form of constitutive equations

$$
\begin{aligned}
& \vec{N}=\mathcal{C}_{N}\left(\vec{\gamma}-\vec{\gamma}_{0}, \vec{\kappa}-\vec{\kappa}_{0}\right) \\
& \vec{M}=\mathcal{C}_{M}\left(\vec{\gamma}-\vec{\gamma}_{0}, \vec{\kappa}-\vec{\kappa}_{0}\right)
\end{aligned}
$$

with operators $\mathcal{C}_{N}$ and $\mathcal{C}_{M}$ being invariant under superimposed rigid-body motions and at least once differentiable with respect to $\vec{\gamma}, \vec{\kappa}$, and $x$, but otherwise arbitrary. $\vec{\gamma}_{0}$ and $\vec{\kappa}_{0}$ are strain vectors of the reference configuration. Consequently, $\vec{N}$ and $\vec{M}$ may be non-zero vectors at the reference configuration.

\section{Generalized virtual work principle}

The rewriting of the virtual work principle (15) for a beam of initial length $L$ gives

$$
\begin{aligned}
\int_{0}^{L}\left(\vec{N} \cdot \delta(\vec{\gamma})_{\text {rel }}+\vec{M} \cdot \delta(\vec{\kappa})_{\text {rel }}\right) d x=\int_{0}^{L}(\vec{n} \cdot \delta \vec{r}+\vec{m} \cdot \delta \vec{\vartheta}) d x \\
+\vec{S}^{0} \cdot \delta \vec{r}(0)+\vec{P}^{0} \cdot \delta \vec{\vartheta}(0)+\vec{S}^{L} \cdot \delta \vec{r}(L)+\vec{P}^{L} \cdot \delta \vec{\vartheta}(L)
\end{aligned}
$$

$\vec{S}^{0}, \vec{P}^{0}, \vec{S}^{L}, \vec{P}^{L}$ are vectors of the external point loads and moments at the boundaries $x=0$ and $x=L . \delta \vec{r}(0), \delta \vec{\vartheta}(0), \delta \vec{r}(L)$ and $\delta \vec{\vartheta}(L)$ are variations of the position vector and the rotational vector at $x=0$ and $x=L$, respectively.

In (30), the strain and the kinematic vectors are related by the kinematic conditions (22)-(23). According to the method of Lagrangian multipliers in 
constrained problems of calculus of variations and the related work of Planinc et al. [24] on planar beams, the constraining kinematic equations

$$
\begin{aligned}
\vec{\gamma}-\vec{r}^{\prime}-\vec{c} & =\overrightarrow{0} \\
\vec{\kappa}-\mathcal{T} \vec{\vartheta}^{\prime}-\vec{d} & =\overrightarrow{0}
\end{aligned}
$$

are scalarly multiplied by arbitrary, independent, vector functions $\vec{a}(x)$ and $\vec{b}(x)$; they must be at least once differentiable on $[0, L]$ except for a finite number of discrete points. The scalar products of the multipliers and the constraining equations are integrated along the length

$$
\begin{array}{r}
\int_{0}^{L} \vec{a} \cdot\left(\vec{\gamma}-\vec{r}^{\prime}-\vec{c}\right) d x=0 \\
\int_{0}^{L} \vec{b} \cdot\left(\vec{\kappa}-\mathcal{T} \vec{\vartheta}^{\prime}-\vec{d}\right) d x=0
\end{array}
$$

and varied with respect to $\vec{a}, \vec{b}, \vec{\gamma}, \vec{\kappa}, \vec{r}$, and $\vec{\vartheta}$

$$
\begin{gathered}
\int_{0}^{L} \delta \vec{a} \cdot\left(\vec{\gamma}-\vec{r}^{\prime}-\vec{c}\right) d x+\int_{0}^{L} \vec{a} \cdot \delta\left(\vec{\gamma}-\vec{r}^{\prime}-\vec{c}\right) d x=0 \\
\int_{0}^{L} \delta \vec{b} \cdot\left(\vec{\kappa}-\mathcal{T} \vec{\vartheta}^{\prime}-\vec{d}\right) d x+\int_{0}^{L} \vec{b} \cdot \delta\left(\vec{\kappa}-\overrightarrow{\mathcal{T}}^{\prime}-\vec{d}\right) d x=0 .
\end{gathered}
$$

The variations in the second integrals are the variations of equations (22) and (23). Their variational forms are given by equations (20) and (21). Thus, we can write

$$
\begin{array}{r}
\int_{0}^{L} \delta \vec{a} \cdot\left(\vec{\gamma}-\vec{r}^{\prime}-\vec{c}\right) d x+\int_{0}^{L} \vec{a} \cdot\left(\delta(\vec{\gamma})_{\mathrm{rel}}-\delta \vec{r}^{\prime}-\delta \vec{\vartheta} \times \vec{r}^{\prime}\right) d x=0 \\
\int_{0}^{L} \delta \vec{b} \cdot\left(\vec{\kappa}-\overrightarrow{\mathcal{T}}^{\prime}-\vec{d}\right) d x+\int_{0}^{L} \vec{b} \cdot\left(\delta(\vec{\kappa})_{\mathrm{rel}}-\delta \vec{\vartheta}^{\prime}\right) d x=0 .
\end{array}
$$

The terms $\vec{a} \cdot \delta \vec{r}^{\prime}$ and $\vec{b} \cdot \delta \vec{\vartheta}^{\prime}$ are partially integrated and the equality $\vec{a}$. $\left(\delta \vec{\vartheta} \times \vec{r}^{\prime}\right)=-\delta \vec{\vartheta} \cdot\left(\vec{a} \times \vec{r}^{\prime}\right)$ is employed. Then we obtain

$$
\begin{aligned}
\int_{0}^{L} \delta \vec{a} \cdot\left(\vec{\gamma}-\vec{r}^{\prime}-\vec{c}\right) d x+\int_{0}^{L} \vec{a} \cdot \delta(\vec{\gamma})_{\mathrm{rel}} d x-\int_{0}^{L} \delta \vec{\vartheta} \cdot\left(\vec{a} \times \vec{r}^{\prime}\right) d x \\
-[\vec{a} \cdot \delta \vec{r}]_{0}^{L}+\int_{0}^{L} \vec{a}^{\prime} \cdot \delta \vec{r} d x=0 \\
\int_{0}^{L} \delta \vec{b} \cdot\left(\vec{\kappa}-\mathcal{T} \vec{\vartheta}^{\prime}-\vec{d}\right) d x+\int_{0}^{L} \vec{b} \cdot \delta(\vec{\kappa})_{\mathrm{rel}} d x \\
-[\vec{b} \cdot \delta \vec{\vartheta}]_{0}^{L}+\int_{0}^{L} \vec{b}^{\prime} \cdot \delta \vec{\vartheta} d x=0
\end{aligned}
$$

After adding equations (35) and (36) to (30) and rearranging the terms, we 
derive

$$
\begin{aligned}
\int_{0}^{L} & \delta(\vec{\gamma})_{\mathrm{rel}} \cdot(\vec{N}-\vec{a}) d x+\int_{0}^{L} \delta(\vec{\kappa})_{\mathrm{rel}} \cdot(\vec{M}-\vec{b}) d x-\int_{0}^{L} \delta \vec{r} \cdot\left(\vec{n}+\vec{a}^{\prime}\right) d x \\
& +\int_{0}^{L} \delta \vec{\vartheta} \cdot\left[-\vec{m}-\vec{b}^{\prime}+\vec{a} \times(\vec{\gamma}-\vec{c})\right] d x \\
& -\int_{0}^{L} \delta \vec{a} \cdot\left(\vec{\gamma}-\vec{r}^{\prime}-\vec{c}\right) d x-\int_{0}^{L} \delta \vec{b} \cdot\left(\vec{\kappa}-\overrightarrow{\mathcal{T}}^{\prime}-\vec{d}\right) d x \\
& +\delta \vec{r}(0) \cdot\left(\vec{S}^{0}+\vec{a}(0)\right)+\delta \vec{\vartheta}(0) \cdot\left(\vec{P}^{0}+\vec{b}(0)\right) \\
& +\delta \vec{r}(L) \cdot\left(\vec{S}^{L}-\vec{a}(L)\right)+\delta \vec{\vartheta}(L) \cdot\left(\vec{P}^{L}-\vec{b}(L)\right)=0
\end{aligned}
$$

Equation (37) represents the modified principle of virtual work in which the variations $\delta(\vec{\gamma})_{\text {rel }}, \delta(\vec{\kappa})_{\text {rel }}, \delta \vec{\vartheta}, \delta \vec{r}, \delta \vec{a}$, and $\delta \vec{b}$ are arbitrary and independent functions, and the variations $\delta \vec{r}^{0}, \overrightarrow{\delta \vartheta}^{0}, \delta \vec{r}^{L}$, and $\overrightarrow{\delta \vartheta}^{L}$ are arbitrary and independent parameters. Because all the coefficients at the independent variations must vanish, the following Euler-Lagrange equations of the three-dimensional beam are obtained:

$$
\begin{aligned}
\vec{N}-\vec{a} & =\overrightarrow{0} \\
\vec{M}-\vec{b} & =\overrightarrow{0} \\
\vec{n}+\vec{a}^{\prime} & =\overrightarrow{0} \\
\vec{m}+\vec{b}^{\prime}-\vec{a} \times \vec{r}^{\prime} & =\overrightarrow{0} \\
\vec{\gamma}-\vec{r}^{\prime}-\vec{c} & =\overrightarrow{0} \\
\vec{\kappa}-\mathcal{T} \vec{\vartheta}^{\prime}-\vec{d} & =\overrightarrow{0}
\end{aligned}
$$

along with the boundary conditions

$$
\begin{array}{ll}
\vec{S}^{0}+\vec{a}(0)=\overrightarrow{0} & \vec{S}^{L}-\vec{a}(L)=\overrightarrow{0} \\
\vec{P}^{0}+\vec{b}(0)=\overrightarrow{0} & \vec{P}^{L}-\vec{b}(L)=\overrightarrow{0} .
\end{array}
$$

Equations (38)-(43) constitute the system of six vector equations for six unknown vector functions $\vec{a}(x), \vec{b}(x), \vec{r}(x), \vec{\vartheta}(x), \vec{\gamma}(x)$, and $\vec{\kappa}(x)$ for a given set of loads, described by $\vec{n}(x), \vec{m}(x), \vec{S}^{0}, \vec{P}^{0}, \vec{S}^{L}$, and $\vec{P}^{L}$. Equations (42) and (43) are kinematic equations given previously. Equations (40) and (41) are the 
force and moment equilibrium conditions (see (13)-(14)). The physical meaning of the Lagrangian multipliers $\vec{a}$ and $\vec{b}$ is obvious from (40)-(41): $\vec{a}(x)$ is the cross-sectional force resultant at point $x ; \vec{b}(x)$ is the cross-sectional moment resultant at point $x \cdot \vec{a}(x)$ and $\vec{b}(x)$ satisfy the equilibrium equations and will hence be referred to as the equilibrium force and moment. We have already introduced the cross-sectional force and moment resultants $\vec{N}$ and $\vec{M}$, which are computed from the strains by the constitutive equations, and will, to point out the difference with the equilibrium force and moment, be termed the constitutive force and moment. Thus, equations (38) and (39) require the equilibrium force and moment vectors to be equal to the constitutive force and moment vectors. We call these requirements the 'consistent equilibrium at the cross-section'. Inequality of equilibrium and constitutive stresses is a common characteristic of standard finite element formulations. It may be a substantial source of error, especially in materially non-linear problems. An application of these important consistency conditions in the elastic-plastic finite element analysis of plane frames is presented in the paper by Vratanar and Saje [33].

\section{Governing equations of the beam}

Equations (38)-(43) can be divided into two sets. Equations (38) and (39) are non-linear algebraic equations. They are solved by iterative, Newton-like methods. The remaining equations of the system, (40)-(43), consist of four first-order ordinary differential equations. Equations (40)-(42) can be, at least formally, satisfied when $\vec{n}, \vec{m}, \vec{\gamma}$, and $\vec{\kappa}$ are the known functions of $x$ :

$$
\begin{aligned}
\vec{a}(x) & =\vec{a}(0)-\int_{0}^{x} \vec{n}(\xi) d \xi \\
\vec{b}(x) & =\vec{b}(0)+\int_{0}^{x}[\vec{a}(\xi) \times(\vec{\gamma}(\xi)-\vec{c}(\xi))-\vec{m}(\xi)] d \xi \\
\vec{r}(x) & =\vec{r}(0)+\int_{0}^{x}(\vec{\gamma}(\xi)-\vec{c}(\xi)) d \xi .
\end{aligned}
$$

The integrated functions are often too complicated for analytical integration to be possible, so the numerical integration methods must be used. The solution of equation (43) can be written in a form of an integral equation

$$
\vec{\vartheta}(x)=\vec{\vartheta}(0)+\int_{0}^{x} \mathcal{T}^{-1}(\vec{\vartheta}(\xi))(\vec{\kappa}(\xi)-\vec{d}(\xi)) d \xi .
$$

Again the numerical solution method must be used, but this time - because the unknown function, $\vec{\vartheta}$, appears in the integrand - a different class of numerical integration methods must be applied. One such class of methods represents 
the Runge-Kutta methods for systems of first-order ordinary differential equations.

The only two remaining unknown functions, $\vec{\kappa}(x)$ and $\vec{\gamma}(x)$, are obtained by the solution of the algebraic equations (38) and (39). When $\vec{\kappa}(x), \vec{\gamma}(x)$, $\vec{n}(x)$, and $\vec{m}(x)$, are given, unknowns $\vec{a}(x), \vec{b}(x), \vec{r}(x)$, and $\vec{\vartheta}(x)$ can be calculated numerically from (46)-(49).

For the fulfilment of the displacement and rotation boundary conditions, strain vectors $\vec{\kappa}(x)$ and $\vec{\gamma}(x)$ must satisfy not only the equations (38) and (39), but also equations (48) and (49), evaluated at the right end $(x=L)$. The complete set of the governing equations of the three-dimensional beam then consists of the algebraic equations (38) and (39)

$$
\begin{aligned}
& \vec{N}(x)-\vec{a}(x)=\overrightarrow{0} \\
& \vec{M}(x)-\vec{b}(x)=\overrightarrow{0}
\end{aligned}
$$

the kinematical boundary conditions at $x=L$ for $\vec{r}$ and $\vec{\vartheta}$

$$
\begin{gathered}
\vec{r}(L)-\vec{r}(0)-\int_{0}^{L}[\vec{\gamma}(x)-\vec{c}(x)] d x=\overrightarrow{0} \\
\vec{\vartheta}(L)-\vec{\vartheta}(0)-\int_{0}^{L} \mathcal{T}^{-1}(\vec{\vartheta}(x))(\vec{\kappa}(x)-\vec{d}(x)) d x=\overrightarrow{0},
\end{gathered}
$$

and the statical boundary conditions (44) and (45)

$$
\begin{gathered}
\vec{S}^{0}+\vec{a}(0)=\overrightarrow{0} \\
\vec{P}^{0}+\vec{b}(0)=\overrightarrow{0} \\
\vec{S}^{L}-\vec{a}(0)+\int_{0}^{L} \vec{n}(x) d x=\overrightarrow{0} \\
\vec{P}^{L}-\vec{b}(0)-\int_{0}^{L}[\vec{a}(x) \times(\vec{\gamma}(x)-\vec{c}(x))-\vec{m}(x)] d x=\overrightarrow{0} .
\end{gathered}
$$

Equations (50)-(57) represent an abstract formulation of the governing equations of the three-dimensional beam where abstract strain vectors $\vec{\gamma}$ and $\vec{\kappa}$ are the only unknown functions of $x$. No limitations on the choice of the coordinate basis have been set so far. For each particular vector variable and operator in (50)-(57), we now choose its most suitable basis. We choose only between the material $(G)$ or the spatial $(g)$ basis. The componential representation is necessary to formulate the numerical solution of the problem and cannot be avoided at this point. An optimal choice of representation seems to be given by Jelenić and Saje [21] and is also used here; equations (50)-(57) then take 
the matrix form

$$
\begin{aligned}
& \boldsymbol{f}_{1}=\mathbf{R} \boldsymbol{N}_{G}(x)-\boldsymbol{a}_{g}(x)=\mathbf{0} \\
& \boldsymbol{f}_{2}=\mathbf{R} \boldsymbol{M}_{G}(x)-\boldsymbol{b}_{g}(x)=\mathbf{0} \\
& \boldsymbol{h}_{1}=\boldsymbol{r}_{g}^{L}-\boldsymbol{r}_{g}^{0}-\int_{0}^{L} \mathbf{R}\left(\gamma_{G}-\boldsymbol{c}_{G}\right) d x=\mathbf{0} \\
& \boldsymbol{h}_{2}=\boldsymbol{\vartheta}_{g}^{L}-\boldsymbol{\vartheta}_{g}^{0}-\int_{0}^{L} \mathbf{T}^{-1}\left(\boldsymbol{\vartheta}_{g}\right) \mathbf{R}\left(\boldsymbol{\kappa}_{G}-\boldsymbol{d}_{G}\right) d x=\mathbf{0} \\
& \boldsymbol{h}_{3}=\boldsymbol{S}_{g}^{0}+\boldsymbol{a}_{g}^{0}=\mathbf{0} \\
& \boldsymbol{h}_{4}=\boldsymbol{P}_{g}^{0}+\boldsymbol{b}_{g}^{0}=\mathbf{0} \\
& \boldsymbol{h}_{5}=\boldsymbol{S}_{g}^{L}-\boldsymbol{a}_{g}^{0}+\int_{0}^{L} \boldsymbol{n}_{g} d x=\mathbf{0} \\
& \boldsymbol{h}_{6}=\boldsymbol{P}_{g}^{L}-\boldsymbol{b}_{g}^{0}-\int_{0}^{L}\left[\boldsymbol{a}_{g} \times \mathbf{R}\left(\boldsymbol{\gamma}_{G}-\boldsymbol{c}_{G}\right)-\boldsymbol{m}_{g}\right] d x=\mathbf{0},
\end{aligned}
$$

where the indices $g$ and $G$ indicate the basis used. Componential equations have sense only if components in an equation are expressed with respect to the same basis; that is why the transformation matrix $\mathbf{R}$ is added in some of the equations (see also equation (4)). Equations (58)-(65), along with the auxiliary relations $(66)-(71)$

$$
\begin{aligned}
\boldsymbol{a}_{g}(x) & =\boldsymbol{a}_{g}^{0}-\int_{0}^{x} \boldsymbol{n}_{g}(\xi) d \xi \\
\boldsymbol{b}_{g}(x) & =\boldsymbol{b}_{g}^{0}+\int_{0}^{x}\left[\boldsymbol{a}_{g}(\xi) \times \mathbf{R}(\xi)\left(\boldsymbol{\gamma}_{G}(\xi)-\boldsymbol{c}_{G}(\xi)\right)-\boldsymbol{m}_{g}(\xi)\right] d \xi \\
\boldsymbol{r}_{g}(x) & =\boldsymbol{r}_{g}^{0}+\int_{0}^{x} \mathbf{R}(\xi)\left(\boldsymbol{\gamma}_{G}(\xi)-\boldsymbol{c}_{G}(\xi)\right) d \xi \\
\boldsymbol{\vartheta}_{g}^{\prime}(x) & =\mathbf{T}^{-1}\left(\boldsymbol{\vartheta}_{g}(x)\right) \mathbf{R}\left(\boldsymbol{\kappa}_{G}(x)-\boldsymbol{d}_{G}(x)\right) \\
\boldsymbol{N}_{G} & =\mathcal{C}_{N}\left(\gamma_{G}-\boldsymbol{\gamma}_{G, 0}, \boldsymbol{\kappa}-\boldsymbol{\kappa}_{G, 0}\right) \\
\boldsymbol{M}_{G} & =\mathcal{C}_{M}\left(\boldsymbol{\gamma}_{G}-\boldsymbol{\gamma}_{G, 0}, \boldsymbol{\kappa}-\boldsymbol{\kappa}_{G, 0}\right) .
\end{aligned}
$$

constitute the complete set of equations of the three-dimensional beam.

\section{Numerical solution of governing equations}

\subsection{Linearization of equations}

Each of equations (58)-(65) defines a functional, dependent on the (primary) unknowns $\boldsymbol{r}_{g}^{0}, \boldsymbol{\vartheta}_{g}^{0}, \boldsymbol{a}_{g}^{0}, \boldsymbol{b}_{g}^{0}, \boldsymbol{r}_{g}^{L}, \boldsymbol{\vartheta}_{g}^{L}, \boldsymbol{\gamma}_{G}(x)$, and $\boldsymbol{\kappa}_{G}(x)$. For the derivation of the linearized form of (58)-(65), the Gâteaux variation of the functionals is needed. Equations are varied at $\boldsymbol{r}_{g}^{0}, \boldsymbol{\vartheta}_{g}^{0}, \boldsymbol{a}_{g}^{0}, \boldsymbol{b}_{g}^{0}, \boldsymbol{r}_{g}^{L}, \boldsymbol{\vartheta}_{g}^{L}, \boldsymbol{\gamma}_{G}(x), \boldsymbol{\kappa}_{G}(x)$ in 'directions' $\delta \boldsymbol{r}_{g}^{0}, \delta \boldsymbol{\vartheta}_{g}^{0}, \delta \boldsymbol{a}_{g}^{0}, \delta \boldsymbol{b}_{g}^{0}, \delta \boldsymbol{r}_{g}^{L}, \delta \boldsymbol{\vartheta}_{g}^{L}, \delta \boldsymbol{\gamma}_{G}(x)$, and $\delta \boldsymbol{\kappa}_{G}(x)$. Note that $\delta \boldsymbol{r}_{g}^{0}$, 
$\delta \boldsymbol{\vartheta}_{g}^{0}, \delta \boldsymbol{a}_{g}^{0}, \delta \boldsymbol{b}_{g}^{0}, \delta \boldsymbol{r}_{g}^{L}, \delta \boldsymbol{\vartheta}_{g}^{L}, \delta \boldsymbol{\gamma}_{G}(x)$, and $\delta \boldsymbol{\kappa}_{G}(x)$, being the variations of the primary unknowns, are arbitrary and independent vectors.

By definition, the variation of one-column matrix is a one-column matrix whose components are the variations of the components of the varied matrix. The basis used for the component representation of the abstract vector is not varied. This is in exact agreement with the definition of the relative variation of an abstract vector when expressed with respect to the material basis:

$$
\begin{aligned}
& \delta(\vec{\gamma})_{\text {rel }}=\delta \gamma_{G 1} \vec{G}_{1}+\delta \gamma_{G 2} \vec{G}_{2}+\delta \gamma_{G 3} \vec{G}_{3} \\
& \delta(\vec{\kappa})_{\mathrm{rel}}=\delta \kappa_{G 1} \vec{G}_{1}+\delta \kappa_{G 2} \vec{G}_{2}+\delta \kappa_{G 3} \vec{G}_{3} .
\end{aligned}
$$

The deduction of the varied equations is greatly simplified if variations of some of the quantities are prepared in advance. Function $\boldsymbol{a}_{g}(x)$ depends on $\boldsymbol{a}_{g}^{0}$ and $\boldsymbol{n}_{g}(x)$. When the loading is deformation-independent, which is the case in the present analysis, $\boldsymbol{n}_{g}(x)$ does not depend on the primary unknowns, and so

$$
\delta \boldsymbol{a}_{g}(x)=\delta \boldsymbol{a}_{g}^{0}
$$

The variation of the rotational vector as the function of the variations of the primary unknowns follows from (21), when expressed with respect to the selected basis for $\boldsymbol{\kappa}$ and $\boldsymbol{\vartheta}$ :

$$
\mathbf{R} \delta \boldsymbol{\kappa}_{G}=\delta \boldsymbol{\vartheta}_{g}^{\prime} .
$$

Integrating (73) with respect to $x$ gives the relation between the variation of the rotational vector and the variations of the primary unknowns

$$
\delta \boldsymbol{\vartheta}_{g}(x)=\delta \boldsymbol{\vartheta}_{g}^{0}+\int_{0}^{x} \mathbf{R}(\xi) \delta \boldsymbol{\kappa}_{G}(\xi) d \xi
$$

By rewriting the variation of rotational operator (12) into the matrix form, we obtain

$$
\delta \mathbf{R}=\delta \boldsymbol{\Theta} \mathbf{R},
$$

which gives, when applied to an arbitrary one-column matrix $\boldsymbol{u}$,

$$
\delta \mathbf{R} \boldsymbol{u}=\delta \boldsymbol{\Theta} \mathbf{R} \boldsymbol{u}=\delta \boldsymbol{\vartheta}_{g} \times \mathbf{R} \boldsymbol{u},
$$

where ' $x$ ' denotes the cross-product between two one-column matrices:

$$
\boldsymbol{v} \times \boldsymbol{u}=\left[\begin{array}{l}
v_{1} \\
v_{2} \\
v_{3}
\end{array}\right] \times\left[\begin{array}{l}
u_{1} \\
u_{2} \\
u_{3}
\end{array}\right]=\left[\begin{array}{l}
v_{2} u_{3}-v_{3} u_{2} \\
v_{3} u_{1}-v_{1} u_{3} \\
v_{1} u_{2}-v_{2} u_{1}
\end{array}\right] .
$$


It is often more practical if the cross-product, $\boldsymbol{v} \times \boldsymbol{u}$, is written as a product of a skew-symmetric matrix, $\mathbf{S}$, and one-column matrix $\boldsymbol{u}$

$$
\boldsymbol{v} \times \boldsymbol{u}=\left[\begin{array}{ccc}
0 & -v_{3} & v_{2} \\
v_{3} & 0 & -v_{1} \\
-v_{2} & v_{1} & 0
\end{array}\right]\left[\begin{array}{l}
u_{1} \\
u_{2} \\
u_{3}
\end{array}\right]=\mathbf{S}(\boldsymbol{v}) \boldsymbol{u} .
$$

Taking into account that $\boldsymbol{v} \times \boldsymbol{u}=-\boldsymbol{u} \times \boldsymbol{v}$ and using the equation (75), we finally get the relation

$$
\delta \mathbf{R} \boldsymbol{u}=-\mathbf{S}(\mathbf{R} \boldsymbol{u}) \delta \boldsymbol{\vartheta}_{g}=-\mathbf{S}(\mathbf{R} \boldsymbol{u}) \delta \boldsymbol{\vartheta}_{g}^{0}-\mathbf{S}(\mathbf{R} \boldsymbol{u}) \int_{0}^{x} \mathbf{R}(\xi) \delta \boldsymbol{\kappa}_{G}(\xi) d \xi
$$

which holds true for any one-column matrix $\boldsymbol{u}$.

Varying $\boldsymbol{b}_{g}(x)$ as expressed by (67), and employing (72) and (76) gives

$$
\begin{aligned}
\delta \boldsymbol{b}_{g}(x)= & \delta \boldsymbol{b}_{g}^{0}+\int_{0}^{x}\left[\delta \boldsymbol{a}_{g} \times \mathbf{R}\left(\boldsymbol{\gamma}_{G}-\boldsymbol{c}_{G}\right)\right] d \xi+\int_{0}^{x}\left[\boldsymbol{a}_{g} \times \delta \mathbf{R}\left(\boldsymbol{\gamma}_{G}-\boldsymbol{c}_{G}\right)\right] d \xi \\
= & \delta \boldsymbol{b}_{g}^{0}+\delta \boldsymbol{a}_{g}^{0} \times \int_{0}^{x} \mathbf{R}\left(\boldsymbol{\gamma}_{G}-\boldsymbol{c}_{G}\right) d \xi-\int_{0}^{x}\left[\boldsymbol{a}_{g} \times \mathbf{S}\left(\mathbf{R}\left(\boldsymbol{\gamma}_{G}-\boldsymbol{c}_{G}\right)\right) \delta \boldsymbol{\vartheta}_{g}^{0}\right] d \xi \\
& -\int_{0}^{x}\left[\boldsymbol{a}_{g} \times \mathbf{S}\left(\mathbf{R}\left(\boldsymbol{\gamma}_{G}-\boldsymbol{c}_{G}\right)\right) \int_{0}^{\xi} \mathbf{R} \delta \boldsymbol{\kappa}_{G} d \eta\right] d \xi \\
= & \delta \boldsymbol{b}_{g}^{0}-\mathbf{S}\left(\int_{0}^{x} \mathbf{R}\left(\boldsymbol{\gamma}_{G}-\boldsymbol{c}_{G}\right) d \xi\right) \delta \boldsymbol{a}_{g}^{0}-\int_{0}^{x} \mathbf{S}\left(\boldsymbol{a}_{g}\right) \mathbf{S}\left(\mathbf{R}\left(\boldsymbol{\gamma}_{G}-\boldsymbol{c}_{G}\right)\right) d \xi \delta \boldsymbol{\vartheta}_{g}^{0} \\
- & \int_{0}^{x} \mathbf{S}\left(\boldsymbol{a}_{g}\right) \mathbf{S}\left(\mathbf{R}\left(\boldsymbol{\gamma}_{G}-\boldsymbol{c}_{G}\right)\right) \int_{0}^{\xi} \mathbf{R} \delta \boldsymbol{\kappa}_{G} d \eta d \xi
\end{aligned}
$$

The linearization of the constitutive equations gives

$$
\begin{aligned}
\delta \boldsymbol{N}_{G} & =\delta \mathcal{C}^{N}=\mathbf{C}_{\gamma \gamma} \delta \boldsymbol{\gamma}_{G}+\mathbf{C}_{\gamma \kappa} \delta \boldsymbol{\kappa}_{G} \\
\delta \boldsymbol{M}_{G} & =\delta \mathcal{C}^{M}=\mathbf{C}_{\kappa \gamma} \delta \gamma_{G}+\mathbf{C}_{\kappa \kappa} \delta \boldsymbol{\kappa}_{G},
\end{aligned}
$$

where the components of matrices $\mathbf{C}_{\gamma \gamma}, \mathbf{C}_{\gamma \kappa}, \mathbf{C}_{\kappa \gamma}$, and $\mathbf{C}_{\kappa \kappa}$ are the partial derivatives of $\mathcal{C}^{N}$ and $\mathcal{C}^{M}$ with respect to $\boldsymbol{\gamma}_{G}(x)$ and $\boldsymbol{\kappa}_{G}(x)$ :

$$
\begin{array}{ll}
\mathbf{C}_{\gamma \gamma}=\left[\frac{\partial \mathcal{C}_{i}^{N}}{\partial \gamma_{j}}\right], & \mathbf{C}_{\gamma \kappa}=\left[\frac{\partial \mathcal{C}_{i}^{N}}{\partial \kappa_{j}}\right] \\
\mathbf{C}_{\kappa \gamma}=\left[\frac{\partial \mathcal{C}_{i}^{M}}{\partial \gamma_{j}}\right], & \mathbf{C}_{\kappa \kappa}=\left[\frac{\partial \mathcal{C}_{i}^{M}}{\partial \kappa_{j}}\right] .
\end{array}
$$

The matrix $\mathbf{C}=\left[\begin{array}{ll}\mathbf{C}_{\gamma \gamma} & \mathbf{C}_{\gamma \kappa} \\ \mathbf{C}_{\kappa \gamma} & \mathbf{C}_{\kappa \kappa}\end{array}\right]$ will be called the cross-section constitutive tangent matrix. 
After these preparations, the variations of the governing equations of the beam are easily derived and are as follows:

$$
\begin{aligned}
\delta \boldsymbol{f}_{1}(x) & =\delta \mathbf{R}(x) \boldsymbol{N}_{G}(x)+\mathbf{R}(x) \delta \boldsymbol{N}_{G}(x)-\delta \boldsymbol{a}_{g}(x) \\
\delta \boldsymbol{f}_{2}(x) & =\delta \mathbf{R}(x) \boldsymbol{M}_{G}(x)+\mathbf{R}(x) \delta \boldsymbol{M}_{G}(x)-\delta \boldsymbol{b}_{g}(x) \\
\delta \boldsymbol{h}_{1} & =\delta \boldsymbol{r}_{g}^{L}-\delta \boldsymbol{r}_{g}^{0}-\int_{0}^{L} \delta \mathbf{R}\left(\boldsymbol{\gamma}_{G}-\boldsymbol{c}_{G}\right) d x-\int_{0}^{L} \mathbf{R} \delta \boldsymbol{\gamma}_{G} d x \\
\delta \boldsymbol{h}_{2} & =\delta \boldsymbol{\vartheta}_{g}^{L}-\delta \boldsymbol{\vartheta}_{g}^{0}-\int_{0}^{L} \mathbf{R} \delta \boldsymbol{\kappa}_{G} d x \\
\delta \boldsymbol{h}_{3} & =\delta \boldsymbol{a}_{g}^{0} \\
\delta \boldsymbol{h}_{4} & =\delta \boldsymbol{b}_{g}^{0} \\
\delta \boldsymbol{h}_{5} & =-\delta \boldsymbol{a}_{g}^{0} \\
\delta \boldsymbol{h}_{6} & =-\delta \boldsymbol{b}_{g}(L) .
\end{aligned}
$$

Equation (83) needs additional comments. Boundary condition (61) is the integral form of the differential equation

$$
\mathbf{T} \vartheta_{g}^{\prime}=\mathbf{R} \kappa_{G},
$$

when the solution is evaluated at $x=L$. Equation (88) represents the matrix form of equation (23). Its variation is given by equation (21). With respect to the selected bases, equation (21) reads

$$
\delta \boldsymbol{\vartheta}_{g}{ }^{\prime}=\boldsymbol{R} \delta \boldsymbol{\kappa}_{G}
$$

By integrating (89) with respect to $x$ and evaluating the result at $x=L$, we obtain the result in (83). The substitution of relations (72), (76), (77), (78), and (79) into (80)-(87) yields the variations of all equations with respect to the variations of the primary unknowns:

$$
\begin{aligned}
\delta \boldsymbol{f}_{1}(x)= & -\mathbf{S}\left(\mathbf{R}(x) \boldsymbol{N}_{G}(x)\right) \delta \boldsymbol{\vartheta}_{g}^{0}-\mathbf{S}\left(\mathbf{R}(x) \boldsymbol{N}_{G}(x)\right) \int_{0}^{x} \mathbf{R}(\xi) \delta \boldsymbol{\kappa}_{G}(\xi) d \xi \\
& +\mathbf{R}(x) \mathbf{C}_{\gamma \gamma}(x) \delta \boldsymbol{\gamma}_{G}(x)+\mathbf{R}(x) \mathbf{C}_{\gamma \kappa}(x) \delta \boldsymbol{\kappa}_{G}(x)-\delta \boldsymbol{a}_{g}^{0} \\
\delta \boldsymbol{f}_{2}(x)= & -\mathbf{S}\left(\mathbf{R}(x) \boldsymbol{M}_{G}(x)\right) \delta \boldsymbol{\vartheta}_{g}^{0}-\mathbf{S}\left(\mathbf{R}(x) \boldsymbol{M}_{G}(x)\right) \int_{0}^{x} \mathbf{R}(\xi) \delta \boldsymbol{\kappa}_{G}(\xi) d \xi \\
& +\mathbf{R}(x) \mathbf{C}_{\kappa \gamma}(x) \delta \boldsymbol{\gamma}_{G}(x)+\mathbf{R}(x) \mathbf{C}_{\kappa \kappa}(x) \delta \boldsymbol{\kappa}_{G}(x)-\delta \boldsymbol{b}_{g}^{0} \\
& +\mathbf{S}\left(\int_{0}^{x} \mathbf{R}\left(\boldsymbol{\gamma}_{G}-\boldsymbol{c}_{G}\right) d \xi\right) \delta \boldsymbol{a}_{g}^{0}+\int_{0}^{x} \mathbf{S}\left(\boldsymbol{a}_{g}\right) \mathbf{S}\left(\mathbf{R}\left(\boldsymbol{\gamma}_{G}-\boldsymbol{c}_{G}\right)\right) d \xi \delta \boldsymbol{\vartheta}_{g}^{0} \\
& +\int_{0}^{x} \mathbf{S}\left(\boldsymbol{a}_{g}\right) \mathbf{S}\left(\mathbf{R}\left(\boldsymbol{\gamma}_{G}-\boldsymbol{c}_{G}\right)\right) \int_{0}^{\xi} \mathbf{R} \delta \boldsymbol{\kappa}_{G} d \eta d \xi \\
\delta \boldsymbol{h}_{1}= & \delta \boldsymbol{r}_{g}^{L}-\delta \boldsymbol{r}_{g}^{0}+\int_{0}^{L} \mathbf{S}\left(\mathbf{R}\left(\boldsymbol{\gamma}_{G}-\boldsymbol{c}_{G}\right)\right) d x \delta \boldsymbol{\vartheta}_{g}^{0} \\
& +\int_{0}^{L} \mathbf{S}\left(\mathbf{R}\left(\boldsymbol{\gamma}_{G}-\boldsymbol{c}_{G}\right)\right) \int_{0}^{x} \mathbf{R} \delta \boldsymbol{\kappa}_{G} d \xi d x-\int_{0}^{L} \mathbf{R} \delta \boldsymbol{\gamma}_{G} d x
\end{aligned}
$$




$$
\begin{aligned}
\delta \boldsymbol{h}_{2}= & \delta \boldsymbol{\vartheta}_{g}^{L}-\delta \boldsymbol{\vartheta}_{g}^{0}-\int_{0}^{L} \mathbf{R} \delta \boldsymbol{\kappa}_{G} d x \\
\delta \boldsymbol{h}_{3}= & \delta \boldsymbol{a}_{g}^{0} \\
\delta \boldsymbol{h}_{4}= & \delta \boldsymbol{b}_{g}^{0} \\
\delta \boldsymbol{h}_{5}= & -\delta \boldsymbol{a}_{g}^{0} \\
\delta \boldsymbol{h}_{6}= & \mathbf{S}\left(\int_{0}^{L} \mathbf{R}\left(\boldsymbol{\gamma}_{G}-\boldsymbol{c}_{G}\right) d x\right) \delta \boldsymbol{a}_{g}^{0}+\int_{0}^{L} \mathbf{S}\left(\boldsymbol{a}_{g}\right) \mathbf{S}\left(\mathbf{R}\left(\boldsymbol{\gamma}_{G}-\boldsymbol{c}_{G}\right)\right) d x \delta \boldsymbol{\vartheta}_{g}^{0} \\
& +\int_{0}^{L} \mathbf{S}\left(\boldsymbol{a}_{g}\right) \mathbf{S}\left(\mathbf{R}\left(\boldsymbol{\gamma}_{G}-\boldsymbol{c}_{G}\right)\right) \int_{0}^{x} \mathbf{R} \delta \boldsymbol{\kappa}_{G} d \xi d x-\delta \boldsymbol{b}_{g}^{0} .
\end{aligned}
$$

\subsection{Discrete form of governing equations}

Although the first variations of the governing equations are linear functionals, they do not appear in the form of a linear combination of the primary unknowns $\delta \boldsymbol{\gamma}_{G}(x)$ and $\delta \boldsymbol{\kappa}_{G}(x)$ (see equations (90)-(93) and (97)). This additional difficulty arises from the multiplicative nature of three-dimensional rotations, which results in the multiplicative formula (12) for the variation of the rotational operator. In order to obtain the linear-combination form for the variations of the rotational operator and, consequently, for the first variations of the governing equations, we must introduce the interpolation of our basic unknowns $\gamma_{G}(x)$ and $\boldsymbol{\kappa}_{G}(x)$.

Functions $\boldsymbol{\gamma}_{G}(x)$ and $\boldsymbol{\kappa}_{G}(x)$ are replaced by a set of their unknown values $\boldsymbol{\gamma}_{G}^{p}$ and $\boldsymbol{\kappa}_{G}^{p}$ at discrete points $x_{p} ; p=1, \ldots, N$, chosen from the interval $[0, L]$, and interpolated by a set of $N$ interpolation functions $I_{p}(x)$

$$
\begin{aligned}
\gamma_{G}(x) & =I_{p}(x) \boldsymbol{\gamma}_{G}^{p} \\
\boldsymbol{\kappa}_{G}(x) & =I_{p}(x) \boldsymbol{\kappa}_{G}^{p}
\end{aligned}
$$

The repeated index $p$ is the summation index. Points $x_{p}$ are called 'interpolation points'. The interpolation as employed in (98)-(99) is mathematically correct because the primary unknown functions $\boldsymbol{\gamma}_{G}(x)$ and $\boldsymbol{\kappa}_{G}(x)$ are additive. This fact substantially simplifies the Newton update procedure (discussed more precisely in the next subsection) because it allows us to introduce the same interpolation for the variations of $\gamma_{G}$ and $\boldsymbol{\kappa}_{G}$

$$
\begin{aligned}
\delta \boldsymbol{\gamma}_{G}(x) & =I_{p}(x) \delta \boldsymbol{\gamma}_{G}^{p} \\
\delta \boldsymbol{\kappa}_{G}(x) & =I_{p}(x) \delta \boldsymbol{\kappa}_{G}^{p}
\end{aligned}
$$

We are now able to rewrite the integrals containing $\delta \boldsymbol{\kappa}_{G}$ and $\delta \boldsymbol{\gamma}_{G}$ as the forms, 
linear in $\delta \boldsymbol{\gamma}_{G}^{p}$ and $\delta \boldsymbol{\kappa}_{G}^{p}(p=1,2, \ldots, N)$ :

$$
\begin{aligned}
\int_{0}^{x} \mathbf{R}(\xi) \delta \boldsymbol{\kappa}_{G}(\xi) d \xi & =\int_{0}^{x} \mathbf{R}(\xi) I_{p}(\xi) \delta \boldsymbol{\kappa}_{G}^{p} d \xi=\int_{0}^{x} I_{p}(\xi) \mathbf{R}(\xi) d \xi \delta \boldsymbol{\kappa}_{G}^{p} \\
\int_{0}^{x} \mathbf{R}(\xi) \delta \boldsymbol{\gamma}_{G}(\xi) d \xi & =\int_{0}^{x} \mathbf{R}(\xi) I_{p}(\xi) \delta \boldsymbol{\gamma}_{G}^{p} d \xi=\int_{0}^{x} I_{p}(\xi) \mathbf{R}(\xi) d \xi \delta \boldsymbol{\gamma}_{G}^{p}
\end{aligned}
$$

By introducing the interpolation for $\boldsymbol{\gamma}_{G}(x)$ and $\boldsymbol{\kappa}_{G}(x)$, we convert a continuum problem into a discrete one, and search a discrete, approximative (interpolated) solution. Instead of unknown functions $\boldsymbol{\gamma}_{G}(x)$ and $\boldsymbol{\kappa}_{G}(x), 2 N$ discrete one-column matrices, $\gamma_{G}^{p}$ and $\boldsymbol{\kappa}_{G}^{p}$, are sought.

Once the unknown functions are discretized, it is also reasonable for the algebraic equations (58)-(59) to be discretized. This is achieved by satisfying equations (58)-(59) at $N$ pre-selected discrete points $x_{q} ; q=1, \ldots, N$, not necessarily coincidental to the interpolation points $x_{p} ; p=1, \ldots, N$. After such a collocation-type discretization is made, a set of $2 N$ matrix equations is obtained, which needs to be solved for $2 N$ unknowns $\boldsymbol{\gamma}_{G}^{p}$ and $\boldsymbol{\kappa}_{G}^{p}(p, q=1$, $\ldots, N)$ :

$$
\begin{aligned}
& \mathbf{R} \boldsymbol{N}_{G}\left(x_{q}\right)-\boldsymbol{a}_{g}\left(x_{q}\right)=\mathbf{0} \\
& \mathbf{R} \boldsymbol{M}_{G}\left(x_{q}\right)-\boldsymbol{b}_{g}\left(x_{q}\right)=\mathbf{0} .
\end{aligned}
$$

The resulting system of discrete governing equations (104)-(105) and (60)(65) constitute a system of $2 N+6$ non-linear matrix equations of a beam element, where $N$ denotes the number of discretization points. The vector unknowns are $\boldsymbol{r}_{g}^{0}, \boldsymbol{\vartheta}_{g}^{0}, \boldsymbol{a}_{g}^{0}, \boldsymbol{b}_{g}^{0}, \boldsymbol{r}_{g}^{L}, \boldsymbol{\vartheta}_{g}^{L}, \boldsymbol{\gamma}_{G}^{p}$, and $\boldsymbol{\kappa}_{G}^{p}(p=1,2, \ldots, N)$.

The use of the discretizations (104)-(105) and the linear forms (102) and (103) enables the Jacobian matrix of the system of discrete equations (104)-(105) and (60)-(65) to be easily obtained

$$
\begin{aligned}
\delta \boldsymbol{f}_{1}\left(x_{q}\right)= & -\mathbf{S}\left(\mathbf{R}\left(x_{q}\right) \boldsymbol{N}_{G}\left(x_{q}\right)\right) \delta \boldsymbol{\vartheta}_{g}^{0}-\mathbf{S}\left(\mathbf{R}\left(x_{q}\right) \boldsymbol{N}_{G}\left(x_{q}\right)\right) \int_{0}^{x_{q}} I_{p}(\xi) \mathbf{R}(\xi) d \xi \delta \boldsymbol{\kappa}_{G}^{p} \\
& +I_{p}\left(x_{q}\right) \mathbf{R}\left(x_{q}\right) \mathbf{C}_{\gamma \gamma}\left(x_{q}\right) \delta \boldsymbol{\gamma}_{G}^{p}+I_{p}\left(x_{q}\right) \mathbf{R}(x) \mathbf{C}_{\gamma \kappa}\left(x_{q}\right) \delta \boldsymbol{\kappa}_{G}^{p}-\delta \boldsymbol{a}_{g}^{0} \\
\delta \boldsymbol{f}_{2}\left(x_{q}\right)= & -\mathbf{S}\left(\mathbf{R}\left(x_{q}\right) \boldsymbol{M}_{G}\left(x_{q}\right)\right) \delta \boldsymbol{\vartheta}_{g}^{0}-\mathbf{S}\left(\mathbf{R}\left(x_{q}\right) \boldsymbol{M}_{G}\left(x_{q}\right)\right) \int_{0}^{x_{q}} I_{p}(\xi) \mathbf{R}(\xi) d \xi \delta \boldsymbol{\kappa}_{G}^{p} \\
& +I_{p}\left(x_{q}\right) \mathbf{R}\left(x_{q}\right) \mathbf{C}_{\kappa \gamma}\left(x_{q}\right) \delta \boldsymbol{\gamma}_{G}^{p}+I_{p}\left(x_{q}\right) \mathbf{R}\left(x_{q}\right) \mathbf{C}_{\kappa \kappa}\left(x_{q}\right) \delta \boldsymbol{\kappa}_{G}^{p}-\delta \boldsymbol{b}_{g}^{0} \\
& +\mathbf{S}\left(\int_{0}^{x_{q}} \mathbf{R}\left(\boldsymbol{\gamma}_{G}-\boldsymbol{c}_{G}\right) d \xi\right) \delta \boldsymbol{a}_{g}^{0}+\int_{0}^{x_{q}} \mathbf{S}\left(\boldsymbol{a}_{g}\right) \mathbf{S}\left(\mathbf{R}\left(\boldsymbol{\gamma}_{G}-\boldsymbol{c}_{G}\right)\right) d \xi \delta \boldsymbol{\vartheta}_{g}^{0} \\
& +\int_{0}^{x_{q}} \mathbf{S}\left(\boldsymbol{a}_{g}\right) \mathbf{S}\left(\mathbf{R}\left(\boldsymbol{\gamma}_{G}-\boldsymbol{c}_{G}\right)\right) \int_{0}^{\xi} I_{p} \mathbf{R} d \eta d \xi \delta \boldsymbol{\kappa}_{G}^{p} \\
\delta \boldsymbol{h}_{1}= & \delta \boldsymbol{r}_{g}^{L}-\delta \boldsymbol{r}_{g}^{0}+\int_{0}^{L} \mathbf{S}\left(\mathbf{R}\left(\boldsymbol{\gamma}_{G}-\boldsymbol{c}_{G}\right)\right) d x \delta \boldsymbol{\vartheta}_{g}^{0} \\
& +\int_{0}^{L} \mathbf{S}\left(\mathbf{R}\left(\boldsymbol{\gamma}_{G}-\boldsymbol{c}_{G}\right)\right) \int_{0}^{x} I_{p} \mathbf{R} \delta \boldsymbol{\kappa}_{G} d \xi d x \delta \boldsymbol{\kappa}_{G}^{p}-\int_{0}^{L} I_{p}(x) \mathbf{R}(x) d x \delta \boldsymbol{\gamma}_{G}^{p}
\end{aligned}
$$




$$
\begin{aligned}
\delta \boldsymbol{h}_{2}= & \delta \boldsymbol{\vartheta}_{g}^{L}-\delta \boldsymbol{\vartheta}_{g}^{0}-\int_{0}^{L} I_{p}(x) \mathbf{R} d x \delta \boldsymbol{\kappa}_{G}^{p} \\
\delta \boldsymbol{h}_{3}= & \delta \boldsymbol{a}_{g}^{0} \\
\delta \boldsymbol{h}_{4}= & \delta \boldsymbol{b}_{g}^{0} \\
\delta \boldsymbol{h}_{5}= & -\delta \boldsymbol{a}_{g}^{0} \\
\delta \boldsymbol{h}_{6}= & \mathbf{S}\left(\int_{0}^{L} \mathbf{R}\left(\boldsymbol{\gamma}_{G}-\boldsymbol{c}_{G}\right) d x\right) \delta \boldsymbol{a}_{g}^{0}+\int_{0}^{L} \mathbf{S}\left(\boldsymbol{a}_{g}\right) \mathbf{S}\left(\mathbf{R}\left(\boldsymbol{\gamma}_{G}-\boldsymbol{c}_{G}\right)\right) d x \delta \boldsymbol{\vartheta}_{g}^{0} \\
& +\int_{0}^{L} \mathbf{S}\left(\boldsymbol{a}_{g}\right) \mathbf{S}\left(\mathbf{R}\left(\boldsymbol{\gamma}_{G}-\boldsymbol{c}_{G}\right)\right) \int_{0}^{x} I_{p} \mathbf{R} d \xi d x \delta \boldsymbol{\kappa}_{G}^{p}-\delta \boldsymbol{b}_{g}^{0} .
\end{aligned}
$$

The matrix coefficients at the variations of the primary unknowns are placed into the Jacobian matrix, which yields

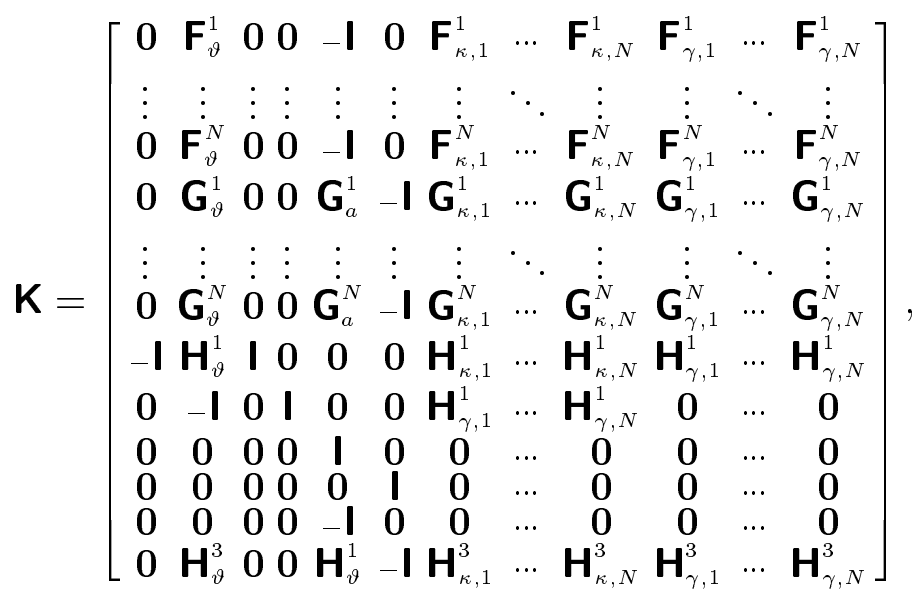

where the $3 \times 3$ block-matrices $\mathbf{F}, \mathbf{G}$ and $\mathbf{H}_{G}$ are defined by

$$
\begin{aligned}
\mathbf{F}_{\vartheta}^{q}= & -\mathbf{S}\left(\mathbf{R} \boldsymbol{N}_{G}\right)_{q} \\
\mathbf{F}_{\kappa, p}^{q}= & \left(I_{p} \mathbf{R} \mathbf{C}_{\gamma \kappa}\right)_{q}-\mathbf{S}\left(\mathbf{R} \boldsymbol{N}_{G}\right)_{q} \int_{0}^{x_{q}} I_{p} \mathbf{R} d \xi \\
\mathbf{F}_{\gamma, p}^{q}= & \left(I_{p} \mathbf{R} \mathbf{C}_{\gamma \gamma}\right)_{q} \\
\mathbf{G}_{\vartheta}^{q}= & -\mathbf{S}\left(\mathbf{R} \boldsymbol{M}_{G}\right)_{q}+\int_{0}^{x_{q}} \mathbf{S}\left(\boldsymbol{a}_{g}\right) \mathbf{S}\left(\mathbf{R}\left(\boldsymbol{\gamma}_{G}-\boldsymbol{c}_{G}\right)\right) d \xi \\
\mathbf{G}_{a}^{q}= & \int_{0}^{x_{q}} \mathbf{S}\left(\mathbf{R}\left(\boldsymbol{\gamma}_{G}-\boldsymbol{c}_{G}\right)\right) d \xi \\
\mathbf{G}_{\kappa, p}^{q}= & \left(I_{p} \mathbf{R} \mathbf{C}_{\kappa \kappa}\right)_{q}-\mathbf{S}\left(\mathbf{R} \boldsymbol{M}_{G}\right)_{q} \int_{0}^{x_{q}} I_{p} \mathbf{R} d \xi \\
& +\int_{0}^{x_{q}} \mathbf{S}\left(\boldsymbol{a}_{g}\right) \mathbf{S}\left(\mathbf{R}\left(\boldsymbol{\gamma}_{G}-\boldsymbol{c}_{G}\right)\right)\left(\int_{0}^{\xi} I_{p} \mathbf{R} d \eta\right) d \xi \\
\mathbf{G}_{\gamma, p}^{q}= & \left(I_{p} \mathbf{R} \mathbf{C}_{\kappa \gamma}\right)_{q}-\int_{0}^{x_{q}} I_{p} \mathbf{S}\left(\boldsymbol{a}_{g}\right) \mathbf{R} d \xi \\
\mathbf{H}_{\vartheta}^{1}= & \int_{0}^{L} \mathbf{S}\left(\mathbf{R}\left(\boldsymbol{\gamma}_{G}-\boldsymbol{c}_{G}\right)\right) d x \\
\mathbf{H}_{\kappa, p}^{1}= & \int_{0}^{L} \mathbf{S}\left(\mathbf{R}\left(\boldsymbol{\gamma}_{G}-\boldsymbol{c}_{G}\right)\right)\left(\int_{0}^{x} I_{p} \mathbf{R} d \xi\right) d x
\end{aligned}
$$




$$
\begin{aligned}
\mathbf{H}_{\gamma, p}^{1} & =-\int_{0}^{L} I_{p} \mathbf{R} d x \\
\mathbf{H}_{\vartheta}^{3} & =\int_{0}^{L} \mathbf{S}\left(\boldsymbol{a}_{g}\right) \mathbf{S}\left(\mathbf{R}\left(\boldsymbol{\gamma}_{G}-\boldsymbol{c}_{G}\right)\right) d x \\
\mathbf{H}_{\kappa, p}^{3} & =\int_{0}^{L} \mathbf{S}\left(\boldsymbol{a}_{g}\right) \mathbf{S}\left(\mathbf{R}\left(\boldsymbol{\gamma}_{G}-\boldsymbol{c}_{G}\right)\right)\left(\int_{0}^{x} I_{p} \mathbf{R} d \xi\right) d x \\
\mathbf{H}_{\gamma, p}^{3} & =-\int_{0}^{L} I_{p} \mathbf{S}\left(\boldsymbol{a}_{g}\right) \mathbf{R} d x .
\end{aligned}
$$

The subscript ' $q$ ' marks that the term is evaluated at $x_{q}$. The column of varied unknowns associated with matrix $\mathbf{K}$ reads

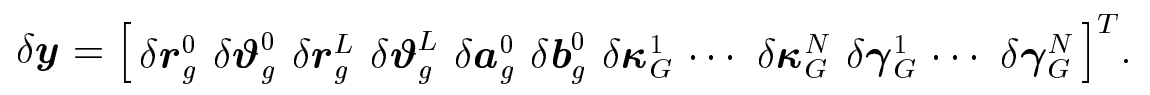

\subsection{Newton's iteration method}

When Newton's method is applied, the non-linearity of the configuration space of the beam requires a special care. The variations of the unknowns are elements of the configuration tangent space. In order to obtain the unknowns, an update must be applied, which maps the unknowns from the tangent to the configuration space. Following Newton's iteration scheme, a system of linear equations is solved at each iteration step $n=0,1,2, \ldots$

$$
\mathbf{K}^{[n]} \delta \boldsymbol{y}=-\boldsymbol{f}^{[n]},
$$

where $\mathbf{K}^{[n]}$ is the global Jacobian tangent matrix, $\boldsymbol{f}^{[n]}$ the residual vector of equations (104)-(105) and (60)-(65), both in iteration $n$, and $\delta \boldsymbol{y}$ a vector of corrections. In classical Newton's method in linear vector spaces, the update is linear which means that $\delta \boldsymbol{y}$ is added to the previous solution iterate vector $\boldsymbol{y}^{[n]}$. The non-linearity of the configuration space requires a non-additive update. Its basic idea is illustrated in Figure 4.

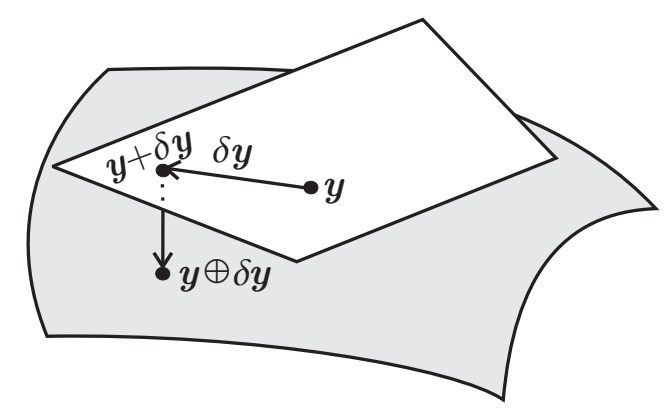

Fig. 4. Update in a non-linear configuration space. 
The non-linear configuration space is symbolically illustrated by a curved surface. The iterative approximation to the solution vector, $\boldsymbol{y}$, is an element of a non-linear space. The correction of the solution vector, $\delta \boldsymbol{y}$, is an element of a linear tangent space; the tangent space is symbolically illustrated by a tangent plane. A new approximation of the solution is obtained first by the determination of a new solution vector on the tangent plane (point $\boldsymbol{y}+\delta \boldsymbol{y}$ ), and then by the projection of the point onto the configuration space.

As a result of an iteration step, the corrections of the unknowns, $\delta \boldsymbol{r}^{0}, \delta \boldsymbol{\vartheta}^{0}$, $\delta \boldsymbol{a}^{0}, \delta \boldsymbol{b}^{0}, \delta \boldsymbol{r}^{L}, \delta \boldsymbol{\vartheta}^{L}, \delta \boldsymbol{\gamma}_{G}^{p}$, and $\delta \boldsymbol{\kappa}_{G}^{p}$, are obtained. As the position vector and stress resultants are linear quantities, their corrections are added to the current iterative approximation values:

$$
\begin{array}{ll}
\boldsymbol{r}_{g}^{0[n+1]}=\boldsymbol{r}_{g}^{0[n]}+\delta \boldsymbol{r}_{g}^{0} & \boldsymbol{r}_{g}^{L[n+1]}=\boldsymbol{r}_{g}^{L[n]}+\delta \boldsymbol{r}_{g}^{L} \\
\boldsymbol{a}_{g}^{0[n+1]}=\boldsymbol{a}_{g}^{0[n]}+\delta \boldsymbol{a}_{g}^{0} & \boldsymbol{b}_{g}^{0[n+1]}=\boldsymbol{b}_{g}^{0[n]}+\delta \boldsymbol{b}_{g}^{0} .
\end{array}
$$

Due to the suitable choice of the basis for the strain vectors, the corrections of strains can be added to the iterative values, too

$$
\begin{aligned}
\gamma_{G}^{p[n+1]} & =\gamma_{G}^{p[n]}+\delta \boldsymbol{\gamma}_{G}^{p} \\
\boldsymbol{\kappa}_{G}^{p[n+1]} & =\boldsymbol{\kappa}_{G}^{p[n]}+\delta \boldsymbol{\kappa}_{G}^{p} .
\end{aligned}
$$

The additivity of strain $\boldsymbol{\gamma}_{G}^{p}$ is obvious, while the additivity of $\boldsymbol{\kappa}_{G}^{p}$ has yet to be proved. Let $\mathcal{R}^{[n]}$ be the rotational operator in iteration $n$ and $\delta \mathcal{R}$ the corresponding update. The total rotation (in iteration $n+1$ ) is then

$$
\mathcal{R}^{[n+1]}=\delta \mathcal{R} \mathcal{R}^{[n]} .
$$

From the total rotation the total operator $\Omega$ is expressed as follows

$$
\begin{aligned}
\Omega^{[n+1]} & =\mathcal{R}^{[n+1]^{\prime}} \mathcal{R}^{[n+1] t}=\left(\delta \mathcal{R} \mathcal{R}^{[n]}\right)^{\prime}\left(\delta \mathcal{R} \mathcal{R}^{[n]}\right)^{t} \\
& =\delta \mathcal{R}^{\prime} \delta \mathcal{R}^{[n] t}+\delta \mathcal{R} \mathcal{R}^{[n] '} \mathcal{R}^{[n] t} \delta \mathcal{R}^{t} \\
& =\delta \Omega+\delta \mathcal{R} \Omega^{[n]} \delta \mathcal{R}^{t} .
\end{aligned}
$$

When applied to an arbitrary abstract vector $\vec{u}$, the above expression can be rewritten as

$$
\Omega^{[n+1]} \vec{u}=\delta \Omega \vec{u}+\delta \mathcal{R} \Omega^{[n]} \delta \mathcal{R}^{t} \vec{u} \quad \rightarrow \quad \vec{\kappa}^{[n+1]} \times \vec{u}=\delta \vec{\kappa} \times \vec{u}+\left(\delta \mathcal{R} \vec{\kappa}^{[n]}\right) \times \vec{u}
$$

From (109) the kinematically exact update of curvature follows, namely

$$
\vec{\kappa}^{[n+1]}=\delta \vec{\kappa}+\delta \mathcal{R}^{[n]} .
$$

Thus, the total curvature in iteration $n+1$ is the sum of the update and the transformed curvature in iteration $n$; the transformation operator in (110) is 
the correction of the rotational operator. This shows that, in general, curvatures (or by analogy, the angular velocities) are not additive. They are additive only if a particular combination of material bases is used. To show this, let us express (110) with respect to the spatial basis

$$
\boldsymbol{\kappa}_{g}^{[n+1]}=\delta \mathbf{R} \boldsymbol{\kappa}_{g}^{[n]}+\delta \boldsymbol{\kappa}_{g}
$$

and then transform the one-column equation with respect to the material basis in iteration $n+1$ :

$$
\begin{aligned}
\boldsymbol{\kappa}_{G}^{[n+1]} & =\left(\mathbf{R}^{[n+1]}\right)^{T} \delta \mathbf{R} \boldsymbol{\kappa}_{g}^{[n]}+\left(\mathbf{R}^{[n+1]}\right)^{T} \delta \boldsymbol{\kappa}_{g} \\
& =\left(\delta \mathbf{R R}^{[n]}\right)^{T} \delta \mathbf{R} \boldsymbol{\kappa}_{g}^{[n]}+\delta \boldsymbol{\kappa}_{G^{[n+1]}} \\
& =\mathbf{R}^{[n] T} \boldsymbol{\kappa}_{g}^{[n]}+\delta \boldsymbol{\kappa}_{G^{[n+1]}} \\
& =\boldsymbol{\kappa}_{G[n]}^{[n]}+\delta \boldsymbol{\kappa}_{G^{[n+1]}} .
\end{aligned}
$$

Formula (4) for the coordinate transformation and $\delta \mathbf{R}^{t} \delta \mathbf{R}=\mathbf{I}$ were taken into account. The result is of a significant importance. When the curvature is expressed with respect to the material basis in iteration $n$, its correction with respect to the material basis in iteration $n+1$ is simply added to it to obtain the total curvature in iteration $n+1$ with respect to the material basis in current iteration $n+1$.

The boundary rotations are, of course, not additive. The update of boundary rotation vectors goes in a standard way. With the Rodrigues formula (8) corrections of boundary rotation matrices, $\delta \mathbf{R}^{0}, \delta \mathbf{R}^{L}$, are first calculated from $\delta \boldsymbol{\vartheta}^{0}$ and $\delta \boldsymbol{\vartheta}^{L}$. Then new boundary rotation matrices are obtained by matrix multiplication:

$$
\begin{aligned}
& \mathbf{R}^{0[n+1]}=\delta \mathbf{R}^{0} \mathbf{R}^{0[n]} \\
& \mathbf{R}^{L[n+1]}=\delta \mathbf{R}^{L} \mathbf{R}^{L[n]}
\end{aligned}
$$

Finally, the Spurrier algorithm [31] is used to extract new boundary rotational vectors $\boldsymbol{\vartheta}^{0[n+1]}$ and $\boldsymbol{\vartheta}^{L[n+1]}$ from $\mathbf{R}^{0[n+1]}$ and $\mathbf{R}^{L[n+1]}$.

The update procedure for $\boldsymbol{\kappa}$ is thus completely additive and will therefore be called additive update. It demands less numerical operations than the multiplicative one and is also more suitable from the theoretical and numerical point of view. When the update is additive, any ordinary additive type of interpolation can be applied in (98) and (99), and the objectivity of such a procedure is not questionable. However, the rotations at $x=L$ obtained by the integration from equation (49) by the use of the curvature vectors, obtained by this procedure, may not be completely equal to the rotations at $x=L$ obtained by the direct nodal update. The error of this additive approach is small, yet it may contribute to the global error of the element. 
When we wish to use an element free of this kind of deficiency, a different update for the curvatures can be employed, here termed the "non-additive update'. It is based on the formula for the variations of rotations, relating the variations of the curvature to the variations of the boundary rotations. Integration of (89) with respect to parameter $x$ gives

$$
\delta \boldsymbol{\vartheta}_{g}(x)=\delta \boldsymbol{\vartheta}_{g}^{0}+\int_{0}^{x} \mathbf{R} \delta \boldsymbol{\kappa}_{G} d \xi
$$

The left hand side in (111), function $\delta \boldsymbol{\vartheta}_{g}$, is the variation of rotational vector which is fully consistent with $\delta \boldsymbol{\vartheta}_{g}^{L}$ at $x=L$. Due to the non-linearity of the configuration space, the corresponding update of curvatures is not the same as if we chose $\delta \boldsymbol{\kappa}_{G}$ as the basic incremental quantity. When $\delta \boldsymbol{\vartheta}_{g}$ is set to be the basic incremental quantity, we need to respect the kinematic equation (23) in order to preserve the kinematic exactness of each incremental solution. The related increment of strain vector, $\Delta \boldsymbol{\kappa}_{G}$, is then obtained by transforming the variation $\delta \boldsymbol{\vartheta}_{g}^{\prime}$ as described in equation (23)

$$
\mathbf{R}^{[n+1]} \Delta \boldsymbol{\kappa}_{G}=\mathbf{T}\left(\delta \boldsymbol{\vartheta}_{g}\right) \delta \boldsymbol{\vartheta}_{g}^{\prime} .
$$

Increment $\Delta \boldsymbol{\kappa}_{G}$ is then added to the current iterative value

$$
\boldsymbol{\kappa}_{G}^{[n+1]}=\boldsymbol{\kappa}_{G}^{[n]}+\Delta \boldsymbol{\kappa}_{G} .
$$

\subsection{Objectivity and path independence of strain measures}

We will now prove, that both strain vectors, when expressed with respect to the material bases, are invariant under a superimposed rigid-body motion. Assume that a rigid-body motion is added to the current configuration $[n]$. The rigid-body rotation matrix, $\mathbf{R}_{R}=$ const, is independent of the arc-length parameter of the beam. It follows that $\mathbf{R}_{R}^{\prime}(x)=\mathbf{0}$ and $\Delta \boldsymbol{\Omega}=\mathbf{R}_{R}^{\prime} \mathbf{R}_{R}^{T}=\mathbf{0}$. The increment of the curvature is then zero as well (see equation (10)):

$$
\Delta \boldsymbol{\kappa}_{G}^{R}(x)=\mathbf{0} .
$$

The rigid-body translation is described by a constant vector $\boldsymbol{r}_{R}=$ const. As $\boldsymbol{r}_{R}^{\prime}$ vanishes, we have (considering equation (22))

$$
\Delta \boldsymbol{\gamma}_{G}^{R}(x)=\mathbf{0} .
$$

The total strains in configuration $[n+1]$ are given by the sums (see $(107)$ and (108))

$$
\begin{aligned}
\boldsymbol{\gamma}_{G}^{[n+1]}(x) & =\boldsymbol{\gamma}_{G}^{[n]}(x)+\Delta \boldsymbol{\gamma}_{G}^{R}=\boldsymbol{\gamma}_{G}^{[n]}(x) \\
\boldsymbol{\kappa}_{G}^{[n+1]}(x) & =\boldsymbol{\kappa}_{G}^{[n]}(x)+\Delta \boldsymbol{\kappa}_{G}^{R}=\boldsymbol{\kappa}_{G}^{[n]}(x) .
\end{aligned}
$$


The fact that the strains remain unchanged, proves the objectivity. This holds if the strain measures are exact. The objectivity need not automatically be conserved after the finite-element discretization, as reported by Crisfield and Jelenić [9]. The non-objectivity of the discrete strain measures is a consequence of the additive-type interpolation used for the rotational parameters. In the present formulation, not the rotations but the strain measures are interpolated. Consequently, the objectivity of discrete strains is preserved automatically. The proof is straightforward. By assumption, functions $\boldsymbol{\gamma}_{G}^{[n]}(x)$ and $\boldsymbol{\kappa}_{G}^{[n]}(x)$ are approximated by

$$
\boldsymbol{\gamma}_{G}^{[n]}(x)=I_{p}(x) \boldsymbol{\gamma}_{G}^{[n]}\left(x_{p}\right) \quad \boldsymbol{\kappa}_{G}^{[n]}(x)=I_{p}(x) \boldsymbol{\kappa}_{G}^{[n]}\left(x_{p}\right) .
$$

The update, given by (107) and (108) or (112), needs then to be applied solely at the discrete interpolation points. If the deformation from $[n]$ to $[n+1]$ is a rigid-body movement, $\Delta \boldsymbol{\kappa}_{G}^{R}$ and $\Delta \boldsymbol{\gamma}_{G}^{R}$ are zero, and the strains at points $x_{p}$ remain unchanged:

$$
\begin{aligned}
\gamma_{G}^{[n+1]}\left(x_{p}\right) & =\gamma_{G}^{[n]}\left(x_{p}\right)+\Delta \boldsymbol{\gamma}_{G}^{R}=\gamma_{G}^{[n]}\left(x_{p}\right) \\
\kappa_{G}^{[n+1]}\left(x_{p}\right) & =\boldsymbol{\kappa}_{G}^{[n]}\left(x_{p}\right)+\Delta \boldsymbol{\kappa}_{G}^{R}=\boldsymbol{\kappa}_{G}^{[n]}\left(x_{p}\right) .
\end{aligned}
$$

These are then directly interpolated

$$
\begin{aligned}
& \boldsymbol{\gamma}_{G}^{[n+1]}(x)=I_{p}(x) \boldsymbol{\gamma}_{G}^{[n+1]}\left(x_{p}\right)=I_{p}(x) \boldsymbol{\gamma}_{G}^{[n]}\left(x_{p}\right)=\boldsymbol{\gamma}_{G}^{[n]}(x) \\
& \boldsymbol{\kappa}_{G}^{[n+1]}(x)=I_{p}(x) \boldsymbol{\kappa}_{G}^{[n+1]}\left(x_{p}\right)=I_{p}(x) \boldsymbol{\kappa}_{G}^{[n]}\left(x_{p}\right)=\boldsymbol{\kappa}_{G}^{[n]}(x),
\end{aligned}
$$

so that the rigid-body motion does not affect the strains at an arbitrary point $x$.

In the present finite-element formulation, the path independence of the strain measures is also straightforward, which is due to the additive type of interpolation. Let us assume that the strains $\boldsymbol{\gamma}_{G}(x)$ and $\boldsymbol{\kappa}_{G}(x)$ have been obtained in two subsequent steps, as $\boldsymbol{\gamma}_{G}^{[1]}(x), \boldsymbol{\gamma}_{G}^{[2]}(x)$ and $\boldsymbol{\kappa}_{G}^{[1]}(x), \boldsymbol{\kappa}_{G}^{[2]}(x)$. As the strains are additive and the addition commutable, the order of the steps is arbitrary:

$$
\begin{aligned}
& \gamma_{G}^{[1+2]}(x)=I_{p}(x)\left(\gamma_{G}^{[1]}\left(x_{p}\right)+\gamma_{G}^{[2]}\left(x_{p}\right)\right)=I_{p}(x)\left(\gamma_{G}^{[2]}\left(x_{p}\right)+\boldsymbol{\gamma}_{G}^{[1]}\left(x_{p}\right)\right)=\gamma_{G}^{[2+1]}(x) \\
& \boldsymbol{\kappa}_{G}^{[1+2]}(x)=I_{p}(x)\left(\boldsymbol{\kappa}_{G}^{[1]}\left(x_{p}\right)+\boldsymbol{\kappa}_{G}^{[2]}\left(x_{p}\right)\right)=I_{p}(x)\left(\boldsymbol{\kappa}_{G}^{[2]}\left(x_{p}\right)+\boldsymbol{\kappa}_{G}^{[1]}\left(x_{p}\right)\right)=\boldsymbol{\kappa}_{G}^{[2+1]}(x) .
\end{aligned}
$$

This proves the path independence of numerical solution. 


\section{$7 \quad$ Numerical examples}

We will present several numerical examples in order to demonstrate the performance and accuracy of the proposed formulation. We will compare our results with other formulations. Linear elastic and concrete material models are employed. For the linear elastic material the operators $\mathcal{C}_{N}$ and $\mathcal{C}_{M}$ in (28)-(29) are taken to be diagonal, so that the relationships between stress resultants and strain measures are given by

$\boldsymbol{N}_{G}=\left[\begin{array}{ccc}E A_{1} & 0 & 0 \\ 0 & G A_{2} & 0 \\ 0 & 0 & G A_{3}\end{array}\right]\left(\boldsymbol{\gamma}_{G}-\boldsymbol{\gamma}_{G, 0}\right) \quad \boldsymbol{M}_{G}=\left[\begin{array}{ccc}G J_{1} & 0 & 0 \\ 0 & E J_{2} & 0 \\ 0 & 0 & E J_{3}\end{array}\right]\left(\boldsymbol{\kappa}_{G}-\boldsymbol{\kappa}_{G, 0}\right)$.

$E$ and $G$ denote elastic and shear moduli of material; $A_{1}$ is the cross-sectional area; $J_{1}$ is the torsional inertial moment of the cross-section; $A_{2}$ and $A_{3}$ are the shear areas in the principal inertial directions $\vec{G}_{2}$ and $\vec{G}_{3}$ of the crosssection; $J_{2}$ and $J_{3}$ are the cross-sectional inertial moments about its principal directions $\vec{G}_{2}$ and $\vec{G}_{3}$.

The arguments of the integrals in equations (104)-(105) and (60)-(65) and, consequently, in the Jacobian matrix, are too complicated for the analytical integration to be possible; therefore, a numerical integration is introduced. We use a classical numerical integration where an integral is replaced by a weighted sum

$$
\int_{0}^{L} f(x) d x \rightarrow \sum_{q=1}^{N} w_{q} f\left(x_{q}\right) .
$$

The values of the weights, $w_{q}$, and the locations of the points, $x_{q}$, are dependent on their number $N$ and on the chosen numerical integration method. Because of its high accuracy, the Gaussian integration is implemented. We assume that the locations of the collocation points, $x_{q}$, as well as the interpolation points, $x_{p}$, coincide with the Gaussian integration points $x_{q}$. Consequently, the Jacobian matrix becomes simpler while no additional interpolation error is introduced into the numerical integrations. Besides the integrals over the whole length of the element, local integrals over a part of the element, $[0, x]$, are also needed in our formulation. For these local integrals, an additional set of local integration points between two global points $x_{q}$ is introduced. In Figure 5 the integration scheme is shown for an element using the 4-point global Gaussian integration $(N=4)$ and the 3-point local Gaussian integration between two global integration points. 


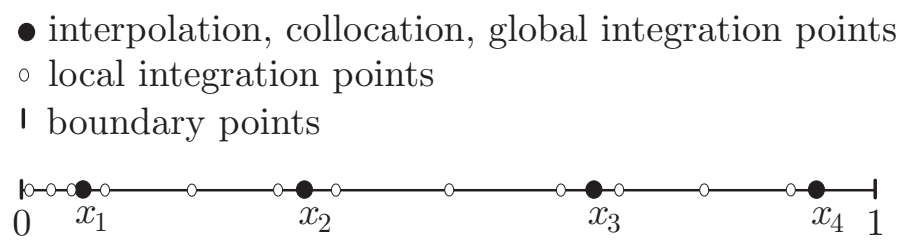

Fig. 5. Interpolation, collocation and global integration points, and local integration points.

It is interesting to observe that the same discrete equations and the Jacobian matrix follow from the Galerkin-type of the finite element method as a special case if the interpolation through the integration points is used. When using the Galerkin finite element method and the interpolation, presented in (100)(101), we obtain $2 N$ integral equations from (37)

$$
\begin{aligned}
& \int_{0}^{L}\left(\mathbf{R} \boldsymbol{N}_{G}-\boldsymbol{a}_{g}\right) I_{p} d x=0 \\
& \int_{0}^{L}\left(\mathbf{R} \boldsymbol{M}_{G}-\boldsymbol{b}_{g}\right) I_{p} d x=0, \quad p=1,2, \ldots, N,
\end{aligned}
$$

which, once replaced by the summations using the numerical integration, yield

$$
\begin{gathered}
\sum_{q=1}^{N} w_{q}\left(\mathbf{R}\left(x_{q}\right) \boldsymbol{N}_{G}\left(x_{q}\right)-\boldsymbol{a}\left(x_{q}\right)\right) I_{p}\left(x_{q}\right)=0 \\
\sum_{q=1}^{N} w_{q}\left(\mathbf{R}\left(x_{q}\right) \boldsymbol{M}_{G}\left(x_{q}\right)-\boldsymbol{b}_{g}\left(x_{q}\right)\right) I_{p}\left(x_{q}\right)=0 .
\end{gathered}
$$

When choosing the interpolation through the integration points, the relation $I_{p}\left(x_{q}\right)=\delta_{p q}$ holds, which results in equations (104)-(105). Thus, the present formulation can be interpreted either as the collocation or the Galerkin method.

Finite elements with various number of interpolation points (taken to coincide with the collocation and integration points) are used in order to investigate the influence of their number on the accuracy of numerical solutions. Because of its minor influence, the effect of the order of the local integration is not displayed in the analysis. A particular element is marked by the symbol ' $E$ ' and equipped with the subscript describing the number, $N$, of interpolation points used. Element $E_{N}$ has thus $6 N+18$ degrees of freedom. Since the nodal values of strains and the internal force and moment vectors at $x=0$ are eliminated at the element level, each element has 12 external degrees of freedom. $E_{2}$, having two interpolation points, was the element of the lowest order used. We wish to stress that the order of interpolation functions is not limited to any particular value in the computer program, so elements of any order can be chosen. 
The local quadratic convergence of Newton's method was achieved in all numerical examples. The iteration was terminated when the Euclidean norm of the vector of nodal unknowns, $\|\delta \boldsymbol{y}\|_{2}$, and of the vector of unbalanced residual forces, $\|\boldsymbol{f}\|_{2}$, was less than $10^{-9}$.

\subsection{Illustration of objectivity}

The following numerical example shows the importance of the correct treatment of the objectivity of the discrete strain measures. Let us discuss first the approach in which the rotations are interpolated. We take that, in iteration $[n]$, the beam is determined by the boundary rotation vectors

$$
\boldsymbol{\vartheta}_{g}^{[n]}(0)=\left[\begin{array}{lll}
1 & -1 & 1
\end{array}\right]^{T} \quad \boldsymbol{\vartheta}_{g}^{[n]}(L)=\left[\begin{array}{lll}
-1 & 1 & 1
\end{array}\right]^{T},
$$

and assume the linear interpolation along the length of the beam

$$
\boldsymbol{\vartheta}_{g}(x)=\boldsymbol{\vartheta}_{g}(0)+\frac{x}{L}\left(\boldsymbol{\vartheta}_{g}(L)-\boldsymbol{\vartheta}_{g}(0)\right) .
$$

Then we have:

$$
\boldsymbol{\vartheta}_{g}^{[n]}\left(\frac{L}{2}\right)=\left[\begin{array}{lll}
0 & 0 & 1
\end{array}\right]^{T}
$$

The rigid rotation, defined by constant rotational vector $\Delta \boldsymbol{\vartheta}_{\mathrm{R}}=\left[\begin{array}{lll}1 & 1 & 1\end{array}\right]^{T}$, is then superimposed onto the current configuration. By multiplying the corresponding rotational matrices and after the use of Spurrier's algorithm for the extraction of the related rotational vectors, we obtain new boundary rotational vectors:

$$
\boldsymbol{\vartheta}_{g}^{[n+1]}(0)=\left[\begin{array}{lll}
2.6372 & 0 & 0.5045
\end{array}\right]^{T} \quad \boldsymbol{\vartheta}_{g}^{[n+1]}(L)=\left[\begin{array}{lll}
0 & 0.5045 & 2.6372
\end{array}\right]^{T} .
$$

Using the linear interpolation (113) for rotations along the beam, we evaluate the rotational vector at the midpoint of the beam from the new boundary values

$$
\boldsymbol{\vartheta}_{g}^{[n+1, \text { interp }]}\left(\frac{L}{2}\right)=\left[\begin{array}{lll}
1.3186 & 0.2522 & 1.5709
\end{array}\right]^{T} .
$$

On the other hand, we can obtain the midpoint rotational vector by the direct superposition of the rigid rotation onto $\boldsymbol{\vartheta}_{g}^{[n]}\left(\frac{L}{2}\right)$

$$
\boldsymbol{\vartheta}_{g}^{[n+1, \mathrm{rot}]}\left(\frac{L}{2}\right)=\Delta \mathbf{R}_{\mathrm{R}} \boldsymbol{\vartheta}_{g}^{[n]}\left(\frac{L}{2}\right)=\left[\begin{array}{lll}
1.5402 & 0.4519 & 1.7976
\end{array}\right]^{T} .
$$

The two vectors differ considerably, which directly affects the rotational strain and, consequently, the internal stresses. Let us now, for simplicity, assume the 
reference configuration of the beam in which the variational constant $\boldsymbol{d}_{G}(x)$ vanishes (this is typical for initially straight beams or beams with a constant curvature). By the use of equation (69) we then evaluate the rotational strain vectors in both configurations. Upon differentiating equation (113) we obtain $\boldsymbol{\vartheta}_{g}^{\prime}(x)=\frac{1}{L}\left(\boldsymbol{\vartheta}_{g}(L)-\boldsymbol{\vartheta}_{g}(0)\right)$ and insert it into (69). The following relation is obtained

$$
\boldsymbol{\kappa}_{G}(x)=\frac{1}{L} \mathbf{R}^{T}\left(\boldsymbol{\vartheta}_{g}(x)\right) \mathbf{T}\left(\boldsymbol{\vartheta}_{g}(x)\right)\left(\boldsymbol{\vartheta}_{g}(L)-\boldsymbol{\vartheta}_{g}(0)\right) .
$$

If we evaluate $\boldsymbol{\kappa}_{G}\left(\frac{L}{2}\right)$ in configurations $[n]$ and $[n+1]$, we observe a considerable difference between the results:

$$
\begin{aligned}
\boldsymbol{\kappa}_{G^{[n]}}^{[n]}\left(\frac{L}{2}\right) & =\frac{1}{L}\left[\begin{array}{lll}
-0.7635 & 2.6023 & 0
\end{array}\right]^{T} \\
\boldsymbol{\kappa}_{G^{[n+1]}}^{[n+1]}\left(\frac{L}{2}\right) & =\frac{1}{L}\left[\begin{array}{lll}
-1.0348 & 2.6182 & 0.4482
\end{array}\right]^{T} .
\end{aligned}
$$

This shows that such an approach is not objective. The difference stems from the application of the additive type of interpolation of rotations which is not correct because the rotations are non-additive quantities. In contrast to the above example, our formulation uses the strain measures as the only interpolated variables. Thus, as analytically proved in section 6.4 , the objectivity of the discrete strain measures is conserved for any type of the interpolation.

\subsection{Lateral buckling of a cantilever. The convergence study}

We consider a straight, inextensible, shear and in plane $(X, Z)$ bending-stiff cantilever, subjected to the point force at its centroid of the free end crosssection (see Figure 6). The lateral, out-of-plane buckling load $F_{c}$ is sought and the numerical results are compared with the analytical solution provided by Timoshenko and Gere [34]. The inextensibility, shear and in-plane bending rigidities were approximated by large values for $G A_{2}, G A_{3}, E A_{1}$, and $E J_{2}$ (Figure 6).

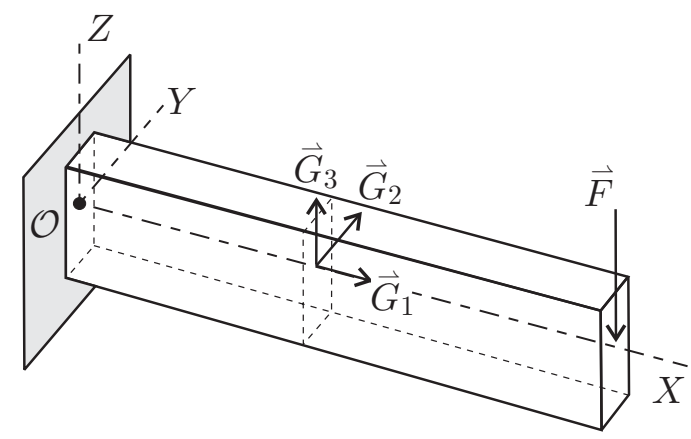

$$
\begin{aligned}
G A_{2} & =G A_{3}=E A_{1}=E J_{2}=10^{15} \\
E J_{3} & =1250, G J_{1}=50, L=100 .
\end{aligned}
$$

Fig. 6. Lateral buckling of a cantilever. 
In Table 1 the influence of the number of elements, the number of interpolation points of an element, and the total number of degrees of freedom on the critical load is displayed. We can see that the increase of the number of interpolation points of an element gives a higher rate of convergence and better accuracy than the increase of the number of elements of equal degree. When a single element with two interpolation points is employed, a substantial error (11.8\%) is found; yet only five elements lead to $0.3 \%$ error. To obtain an eleven-digit accurate solution, 50 elements with 3 points are required; such a mesh has 1506 external plus internal degrees of freedom. Equally accurate results are obtained if one 10-point element with 78 degrees of freedom is used.

Table 1

Out-of-plane buckling load.

\begin{tabular}{|c|c|c|c|c|c|}
\hline$E$ d.o.f. & $n_{e}=1$ & $n_{e}=2$ & $n_{e}=5$ & $n_{e}=10$ & $n_{e}=50$ \\
\hline$E_{2}$ & 0.11221899817 & 0.10143235609 & 0.10034943670 & 0.10031720870 & 0.10031501133 \\
\hline$E_{3} 1506$ & 0.10137598589 & 0.10034917035 & 0.10031516901 & 0.10031498664 & 0.10031498359 \\
\hline$E_{4} \quad 366$ & 0.10040668895 & 0.10031588043 & 0.10031498449 & 0.10031498359 & \\
\hline$E_{5} \quad 216$ & 0.10032093587 & 0.10031500491 & 0.10031498359 & & \\
\hline$E_{6}$ & 0.10031540713 & 0.10031498389 & 0.10031498359 & & \\
\hline$E_{7} \quad 114$ & 0.10031500049 & 0.10031498359 & & & \\
\hline$E_{8}$ & 0.10031498425 & & & & \\
\hline$E_{9}$ & 0.10031498362 & & & & \\
\hline$E_{10} \quad 78$ & 0.10031498359 & & & & \\
\hline
\end{tabular}

$E=$ type of element, $n_{e}=$ number of elements, d.o.f.=total number of degrees of freedom

The accuracy of the numerical solution of two and three-point elements when increasing the number of elements is shown in Figure 7a. The relation between the number of elements and the absolute error of the numerical solution is approximately linear in the logarithmic scale. Thus, the error decreases exponentially with the number of elements

$$
\operatorname{err}\left(n_{e}\right) \approx\left(\frac{1}{n_{e}}\right)^{\alpha}
$$

For linear elements $E_{2}$, the estimated value for $\alpha$ equals 3.6. For quadratic elements $E_{3}$, the convergence rate is substantially higher, $\alpha$ now being approximately 5.4. A notably more rapid convergence is obtained by increasing the order of elements, $N$, while keeping the number of elements fixed, as shown in Figure $7 \mathrm{~b}$. The absolute error of element $E_{N}$ is roughly

$$
\operatorname{err}(N) \approx\left(\frac{1}{N}\right)^{\beta N}
$$


The convergence-rate factor $\beta$ is approximately 0.9 and 3.3 for the single and two-element meshes, respectively.

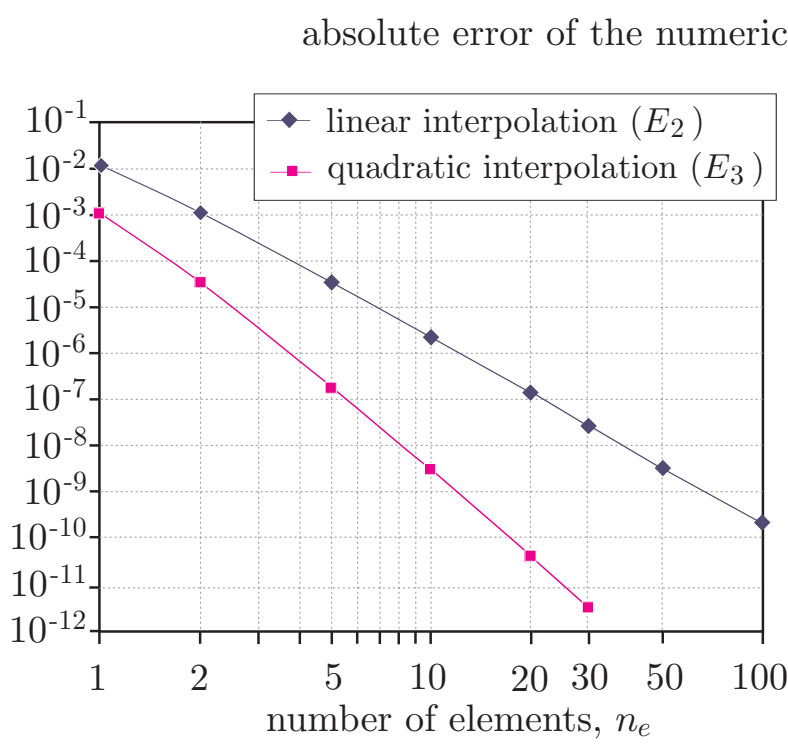

a)

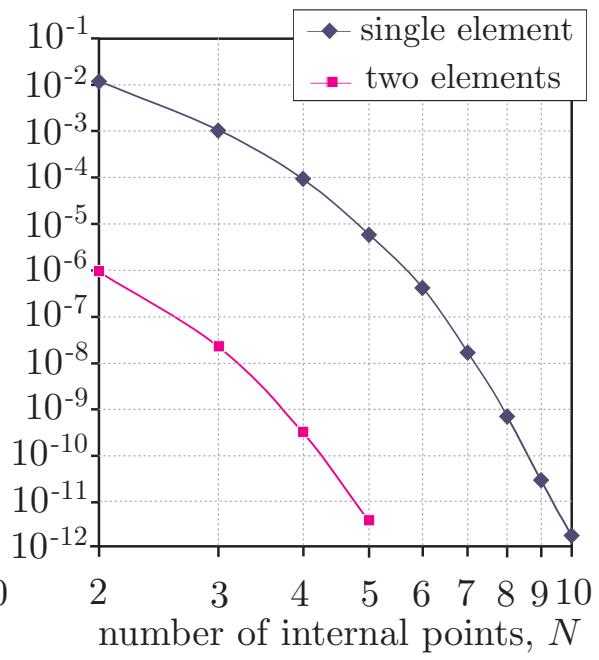

b)

Fig. 7. Absolute error of the numerical solution as a function of a) the number of elements, and b) the number of internal points.

By the increase of the number of elements and/or the number of interpolation points of the element, the complexity of the problem raises. For this reason the convergence needs also to be analyzed with regard to the total number of degrees of freedom. The general behaviour is presented in Figure 8 , where the absolute error is drawn as a surface in the logarithmic scale and the corresponding discrete-point values of degrees of freedom are shown as the three-dimensional bars. Not only the error of the numerical solution decreases more rapidly when the number of interpolation points is increased, but also the growth of the number of degrees of freedom is lower compared to the case where the number of elements is increased. The rate of convergence obtained by increasing the number of interpolation points is even more favourable if we compare the number of floating point operations needed for the execution of the crucial parts of the algorithm: i) the generation of the tangent stiffness matrix and the residual vector of each element and ii) the solution of the global system of linear equations. Since the internal interpolation degrees of freedom are condensed at the element level, the order and the sparsity of the global system of linear equations are independent of the increase of the interpolation points. That is why we also count the number of floating point operations needed for condensation and decondensation of each element. The approximative number of floating point operations needed for the solution of the global system of linear equation is shown in Table 2. In this numerical ex- 


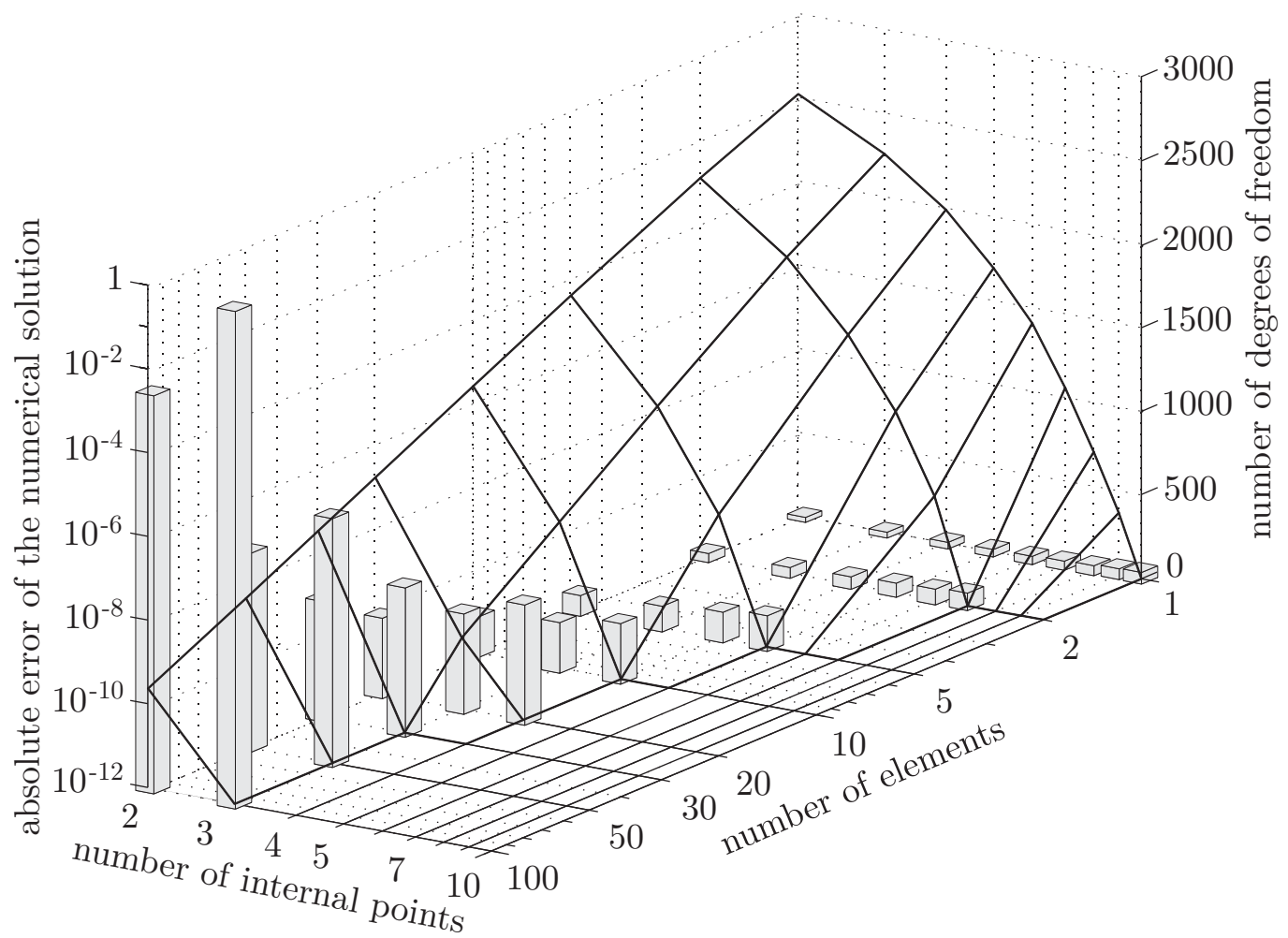

Fig. 8. Convergence of the numerical solution and the corresponding increase in the total number of degrees of freedom.

ample the solution of the global system of equations is not a demanding task, due to a block-diagonal form of the global tangent stiffness matrix. For more complicated structures the block-diagonal form may not be preserved, which would increase the number of operations needed. On the other hand the number of floating point operations at the element level remains approximately the same for any problem. It depends on the number of internal degrees of freedom, $N$, an also on the number of local Gaussian integration points. In Table 3 the approximative number of floating point operations as a function of $N$ is shown for the elements using 3-point local Gaussian integration between the global integration points.

Table 2

Number of floating point operations needed for the solution of the global system of linear equation.

\begin{tabular}{lcccccccc}
\hline$n_{e}$ & 1 & 2 & 5 & 10 & 20 & 30 & 50 & 100 \\
e.d.o.f. & 12 & 18 & 36 & 66 & 126 & 186 & 306 & 606 \\
\hline flops & 100 & 500 & 2100 & 4800 & 10100 & 15000 & 22600 & 46000 \\
\hline
\end{tabular}

$n_{e}=$ number of elements, e.d.o.f.=number of external degrees of freedom, flops $=$ number of floating point operations 
Table 3

Number of floating point operations at the element level.

\begin{tabular}{lccccccccc}
\hline$N$ & 2 & 3 & 4 & 5 & 6 & 7 & 8 & 9 & 10 \\
\hline K and $\boldsymbol{f}$ & 26000 & 38000 & 51000 & 67000 & 87000 & 110000 & 137000 & 166000 & 198000 \\
condensation & 6000 & 8500 & 12000 & 16000 & 22000 & 29000 & 38000 & 49000 & 62000 \\
decondensation & 1000 & 1500 & 2500 & 3500 & 5000 & 7500 & 10000 & 13500 & 17500 \\
\hline total & 33000 & 48000 & 65500 & 86500 & 114000 & 146500 & 185000 & 228500 & 277500 \\
\hline
\end{tabular}

$N=$ number of internal degrees of freedom, $\mathbf{K}$ and $\boldsymbol{f}=$ Jacobian matrix and residual vector

When counting the total number of floating point operations we must multiply the number of elements and the floating point operations at the element level and add it to the floating point operations of the global level. We increased the number of internal points for each finite element mesh to obtain the elevendigit accurate solution. In Figure 9 we display the corresponding number of floating point operations. It is evident that the lowest number of operations is needed when a single element with 10 internal points is used. By the increase of the number of elements the total number of operations increases rapidly which results in higher computational times.

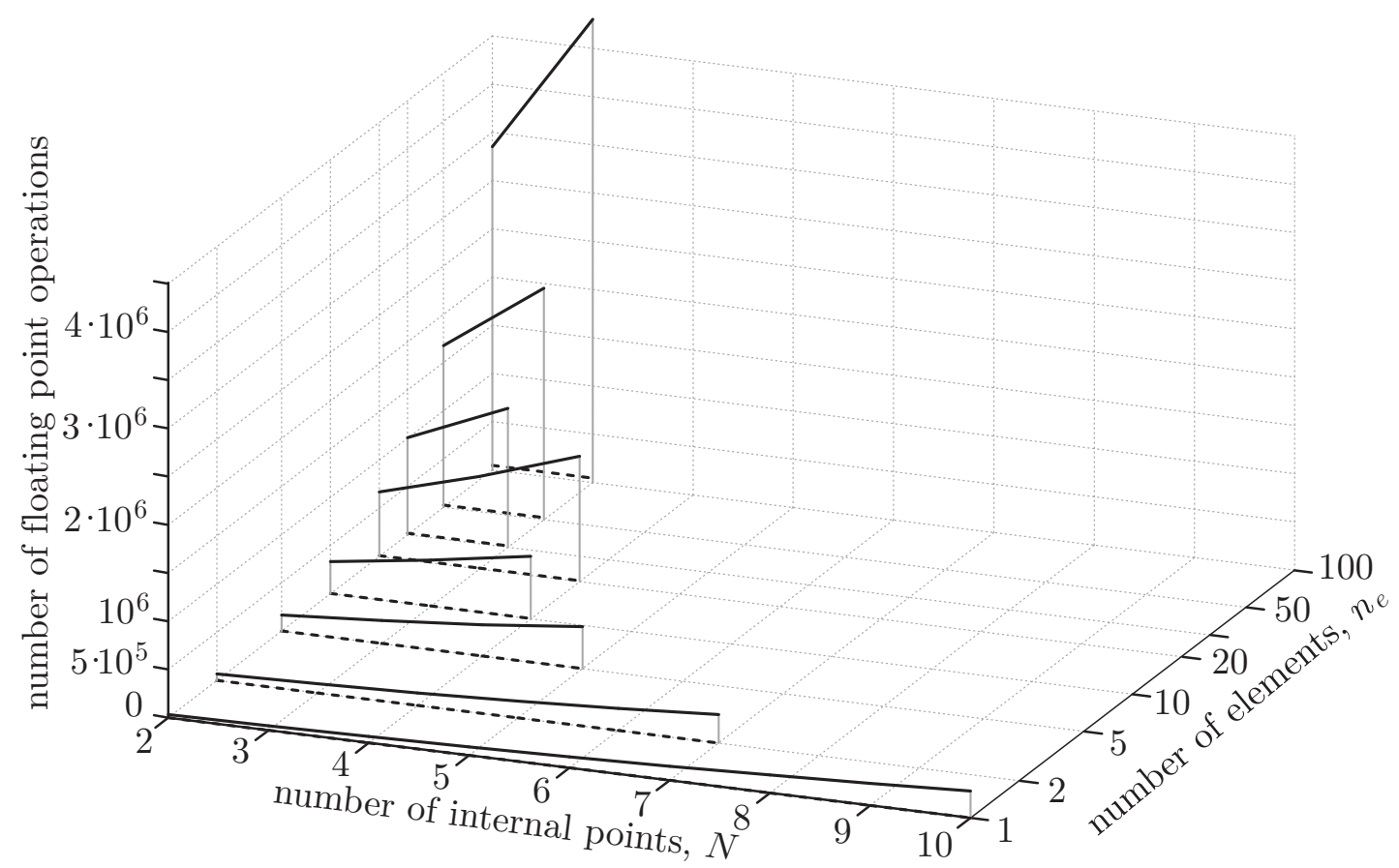

Fig. 9. Total number of floating point operations needed at the element level and for the solution of the global system of equations.

We should point out the complete agreement of our results to those presented by Jelenić and Saje [21]. In the present formulation the number of degrees of 
freedom of an element is larger, due to interpolation of two vector unknowns, $\boldsymbol{\gamma}_{G}$ and $\boldsymbol{\kappa}_{G}$, compared to only one in [21]. The degree of interpolation of a particular vector here suffices to be one degree smaller than in [21] to achieve an equal precision.

\subsection{Lateral buckling of a right-angle frame}

In this classical problem, first introduced by Argyris et al. [2] and studied by many authors (e.g. [21], [23], [29]), we analyze a simply-supported thin rectangular right-angle frame. The undeformed line of centroids lies in the $X Y$-plane. The support at one end allows the translation in the $X$-direction and the rotation about the $Z$-direction. The support on the other end allows only the rotation about the $Z$-direction. The frame is subjected to the in-plane moments, as shown in Figure 10.

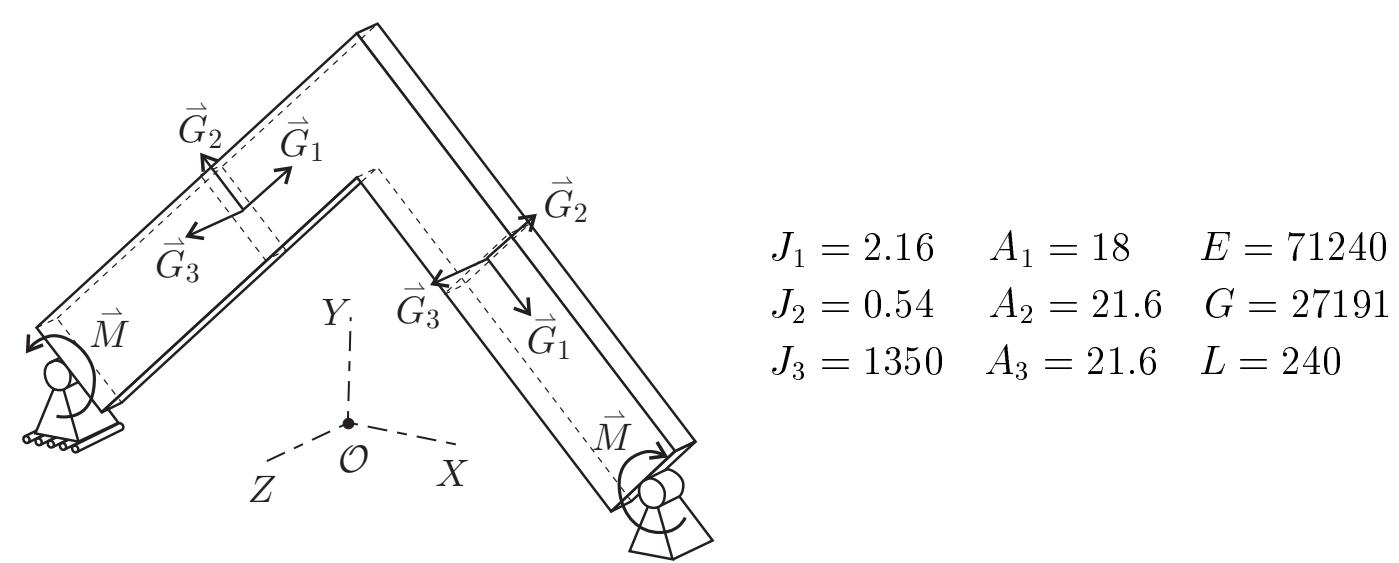

Fig. 10. Lateral buckling of right-angle frame.

Due to the extreme slenderness of the rectangular cross-section (the thickness to height ratio is taken to be $1 / 50$ ), the frame buckles out of the plane. The buckling moment $M_{c}$ is sought by the use of different-order elements and several finite-element meshes. In Table 4 our results are shown, together with their comparison to the analytical solution provided by Timoshenko and Gere [34] and numerical results by other authors.

The four-digit accurate critical moment was obtained by a single element with 6 interpolation points ( 54 degrees of freedom) or by the mesh of 3 elements with 3 interpolation points (108 degrees of freedom). From Table 4 again a rapid convergence of the numerical solution can be observed when increasing the number of interpolation points. The comparison to other authors shows that only the solution by Jelenić and Saje [21] gives the results of equal precision. With regard to the number of degrees of freedom used, the present element is relatively inexpensive for the high accuracy of the solution obtained. 
Table 4

Critical moment $M_{c}$ of the simply supported right-angle frame.

\begin{tabular}{|c|c|c|c|c|c|c|c|c|}
\hline$E$ & d.o.f. & $n_{e}=1$ & $n_{e}=2$ & $n_{e}=3$ & $n_{e}=4$ & $n_{e}=5$ & $n_{e}=6$ & $n_{e}=10$ \\
\hline$E_{2}$ & & \pm 685.99 & \pm 626.88 & \pm 623.20 & \pm 622.54 & \pm 622.35 & \pm 622.29 & \\
\hline$E_{3}$ & 126 & \pm 626.31 & \pm 622.31 & \pm 622.23 & \pm 622.22 & & & \\
\hline$E_{4}$ & 78 & \pm 622.40 & \pm 622.22 & \pm 622.22 & & & & \\
\hline$E_{5}$ & & \pm 622.23 & \pm 622.22 & & & & & \\
\hline$E_{6}$ & 54 & \pm 622.22 & & & & & & \\
\hline$E_{8}$ & & \pm 622.22 & & & & & & \\
\hline [21] & 27 & \pm 622.2 & & & & & & \\
\hline [23] & 66 & & & & & & & \pm 626.7 \\
\hline [29] & 126 & & & & & & & \pm 626 \\
\hline anal & tical sc & ution & 622.21 & & & & & \\
\hline
\end{tabular}

$E=$ type of element, $n_{e}=$ number of elements, d.o.f.=number of degrees of freedom

\subsection{In-plane stability of a deep circular arch}

This example shows the ability of the present elements to capture the stability load at a highly deformed configuration. The in-plane buckling stability is considered of an elastic beam with the centroidal axis shaped as the circular arc, corresponding to the central angle $215^{\circ}$ of a circle with radius $R=100$.

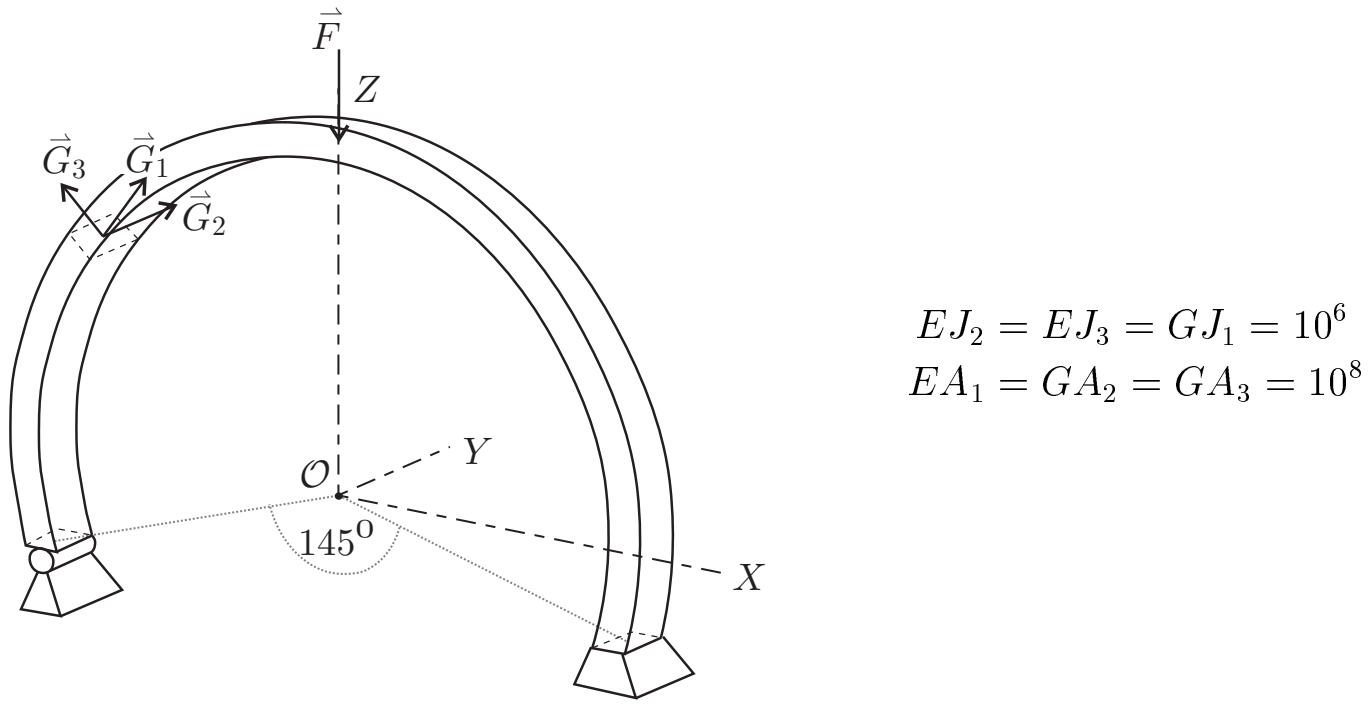

Fig. 11. Deep circular arch. 
The problem has been widely studied. The result of DaDeppo and Schmidt [11] will serve as a reference solution. As estimated in [11], the error of their finite-difference solution is roughly $0.03 \%$, thus the results given in [11] are correct to three digits $\left(F_{c r}=897\right)$. In our study we have chosen the global coordinate system $(X, Y, Z)$, in which the undeformed centroidal axis lies in the plane $X Z$ and the reference point $(0,0,0)$ coincides with the center of the circle. The concentrated force $\vec{F}=-F \vec{g}_{3}$ acts at the point $(0,0, R)$. The remaining values of the material and geometrical parameters are shown in Figure 11. The comparison between various results is presented in Table 5 .

Table 5

Critical force of a deep circular arch.

\begin{tabular}{|c|c|c|c|c|c|c|c|c|c|}
\hline$n_{e}$ & $\begin{array}{r}E_{2} \\
\text { curved }\end{array}$ & $\begin{array}{r}E_{3} \\
\text { curved }\end{array}$ & $\begin{array}{r}E_{5} \\
\text { curved }\end{array}$ & $\begin{array}{r}E_{2} \\
\text { straight }\end{array}$ & $\begin{array}{r}E_{3} \\
\text { straight }\end{array}$ & $\begin{array}{r}{[26]} \\
\text { curved }\end{array}$ & $\begin{array}{r}{[18]} \\
\text { curved }\end{array}$ & $\begin{array}{r}{[18]} \\
\text { straight }\end{array}$ & $\begin{array}{r}{[29]} \\
\text { straight }\end{array}$ \\
\hline 2 & & & 895.91 & & & 904.53 & & & \\
\hline 4 & & & *897.39 & & & * 897.29 & & & \\
\hline 6 & 903.22 & 897.74 & 897.29 & & & & & & \\
\hline 12 & 897.71 & * 897.30 & 897.29 & & & & & & \\
\hline 20 & & & & ${ }^{*} 907.31$ & ${ }^{*} 907.30$ & & *897.5 & ${ }^{*} 906$ & \\
\hline 24 & *897.32 & 897.29 & & & & & & & \\
\hline 40 & & & & 899.80 & 899.80 & & & & ${ }^{*} 905.28$ \\
\hline 48 & 897.29 & 897.29 & & & & & & & \\
\hline d.o.f. & 582 & 366 & 186 & 485 & 606 & 66 & 246 & 246 & 246 \\
\hline e.d.o.f. & 150 & 78 & 30 & 126 & 126 & 30 & 126 & 126 & 246 \\
\hline
\end{tabular}

The results of the present formulation and the results from [26] are found to be the most accurate, while employing the least number of elements. The efficiency of these two formulations also results from the accurate consideration of the initial curvature of the elements. This is confirmed in the fourth and fifth column of Table 5, where you can see the results of the polygonal approximation of the arc for initially straight elements $E_{2}$ and $E_{3}$. The present results are obtained by the use of several types of elements, while other studies use 9-point [26], 3-point [18] and linear finite elements [29]. It is interesting to compare the total and the external number of degrees of freedom used. A particularly low level of the number of degrees of freedom is needed in [26], however a plane model of the beam was used. By the use of 5-point curved element we needed 186 total degrees of freedom, which is the lowest value, comparing only spatial beam theories. For the other types of our elements, the total number of degrees of freedom is higher than in [18] and [29], while 
our formulation needs less external degrees of freedom (i.e. a smaller size of the global tangent stiffness matrix). Note also that if the arc is approximated by a polygonal line, the order of our elements practically does not affect the result.

Both update procedures mentioned in Section 6.3 give the same results in all three presented numerical examples, because we have not been dealing with the out-of-plane rotations. From that point of view, the forthcoming examples are more interesting.

\subsection{Out-of-plane buckling of a cantilever right-angle frame}

The right-angle frame is now clamped at one end and subjected to the point forces at its free end, as shown in Figure 12a. Force $F$ in $X$-direction is ascendant with the largest value taken to be 1.485 . To obtain the post-buckling behaviour, a small perturbation force $F_{p}=0.001 F$ in the out-of-plane direction is applied. The results are obtained by using 100 load steps. The final deformed centroidal axis is shown in Figure $12 \mathrm{~b}$ together with its projection on the $X Z$-plane.
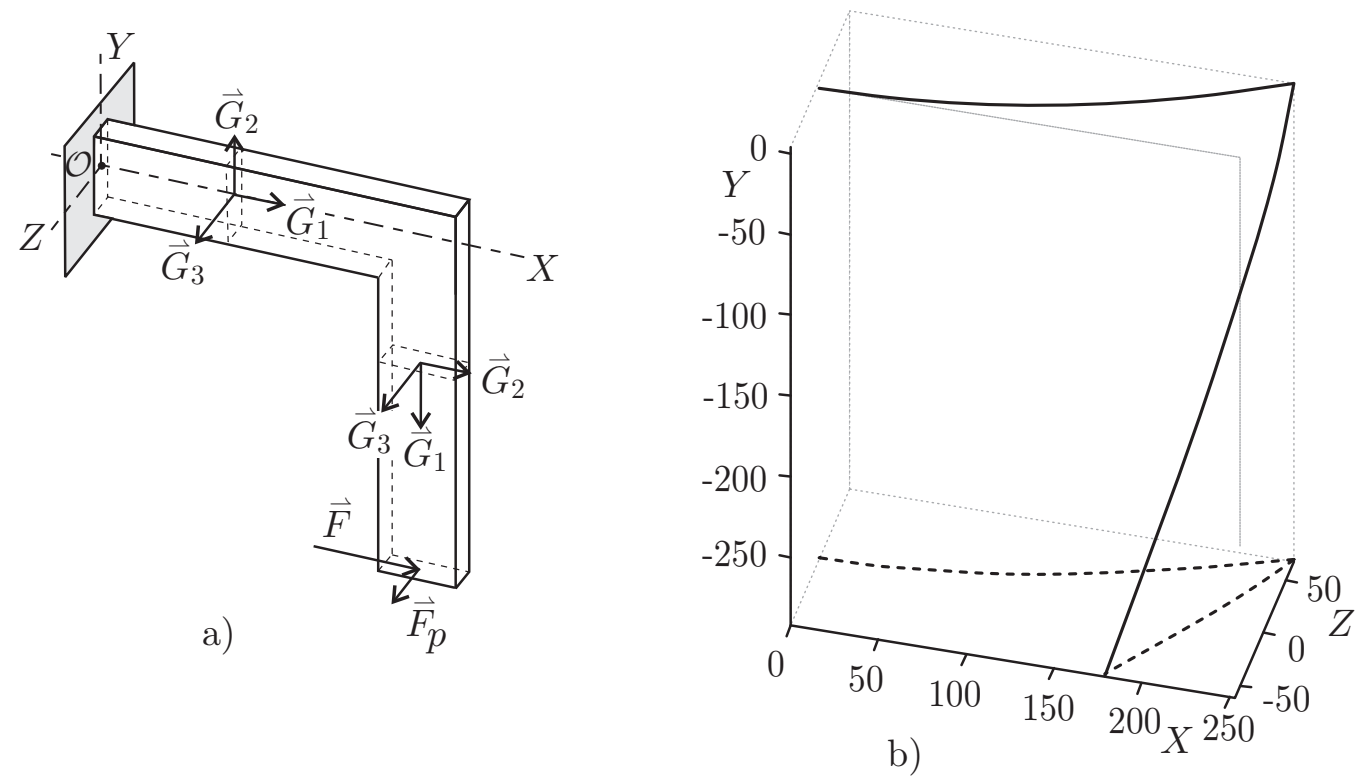

Fig. 12. Lateral buckling of a cantilever right-angle frame: a) the initial configuration, and b) the final deformed centroidal axis.

The results were obtained by using both update procedures, and the forcedisplacement diagrams are compared in Figure 13. The two graphs almost agree. The results in Figure 13, obtained by the mesh of 12 elements with 5 
interpolation points per element, are in agreement with the results of other researchers [5], [29], [30].

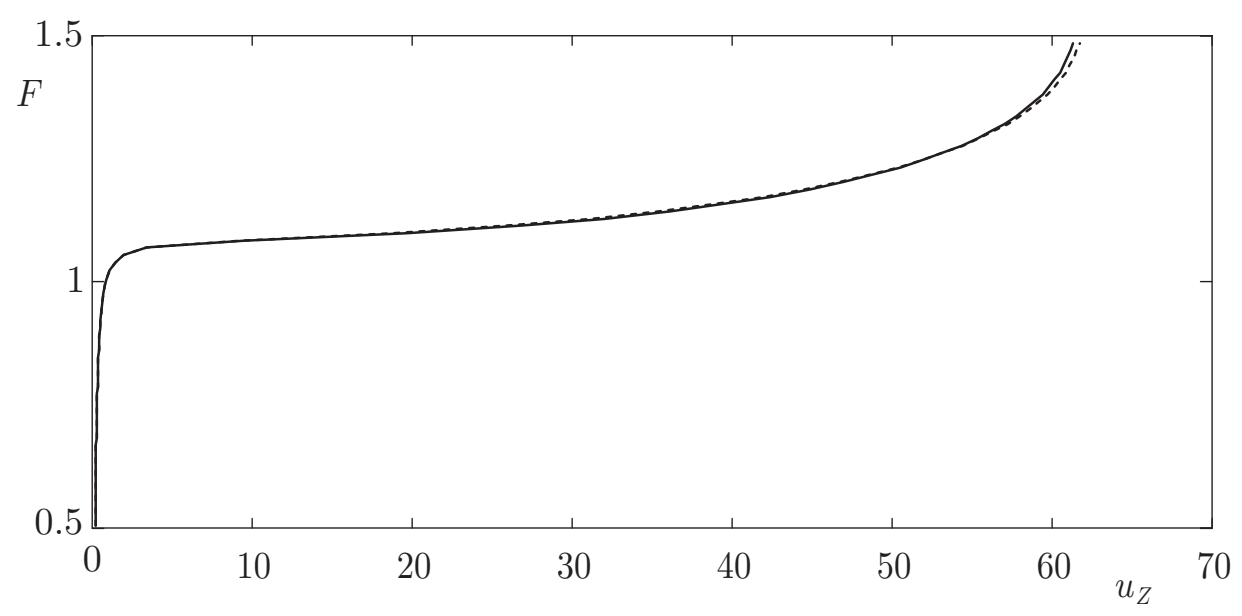

Fig. 13. Out-of-plane displacement versus in-plane force diagram (additive update - dashed line, non-additive update - solid line).

When the additive update is employed, the equation for the rotational vector at $x=L$

$$
\boldsymbol{\vartheta}_{g}^{L}=\boldsymbol{\vartheta}_{g}^{0}-\int_{0}^{L} \mathbf{T}^{-1}\left(\boldsymbol{\vartheta}_{g}\right) \mathbf{R}\left(\boldsymbol{\kappa}_{G}-\boldsymbol{d}_{G}\right) d x
$$

will not give the same value for the right-boundary rotation $\boldsymbol{\vartheta}_{g}^{L}$ as the direct update (see the discussion in Section 6.3). In Table 6 we display the relative error

$$
\frac{\left\|\boldsymbol{\vartheta}_{g}^{L}-\boldsymbol{\vartheta}_{g}^{0}+\int_{0}^{L} \mathbf{T}^{-1}\left(\boldsymbol{\vartheta}_{g}\right) \mathbf{R}\left(\boldsymbol{\kappa}_{G}-\boldsymbol{d}_{G}\right) d x\right\|_{2}}{\left\|\boldsymbol{\vartheta}_{g}^{L}\right\|_{2}}
$$

for each element in the mesh. The error is considerable and spans from roughly $10^{-3}$ to $10^{-5}$. Yet it does not substantially influence the results for the remaining variables. Note also that this error vanishes completely if the non-additive update is used.

Table 6

\begin{tabular}{|c|c|c|c|c|c|c|c|c|c|c|c|}
\hline 1 & 2 & 3 & 4 & 5 & 6 & 7 & 8 & 9 & 10 & 11 & 12 \\
\hline$\varepsilon 8 \cdot 10^{-5}$ & $3 \cdot 10^{-4}$ & $5 \cdot 10^{-4}$ & $7 \cdot 10^{-4}$ & $9 \cdot 10^{-4}$ & $1 \cdot 10^{-3}$ & $1 \cdot 10^{-3}$ & $1 \cdot 10^{-3}$ & $9 \cdot 10^{-4}$ & $7 \cdot 10^{-4}$ & $4 \cdot 10^{-4}$ & $2 \cdot 10^{-4}$ \\
\hline
\end{tabular}

Relative error of the right-boundary rotation in the finite-element mesh. 


\subsection{Twisted cantilever beam}

An initially twisted beam was presented by MacNeal and Harder [22] as one of standard test problems to test finite-element accuracy. The beam is clamped at one end and loaded with unit in-plane and out-of-plane force at the other. The centroidal axis is straight at the beginning, but the cross-sections are twisted about the centroidal axis, as shown in Figure 14. The initial twist angle is taken as a linear function of the arc-length $x$ with its value set to 0 at the clamped end and to $\frac{1}{2} \pi$ at the free end of the beam. The remaining geometric and material characteristics of the beam are shown in Figure 14.

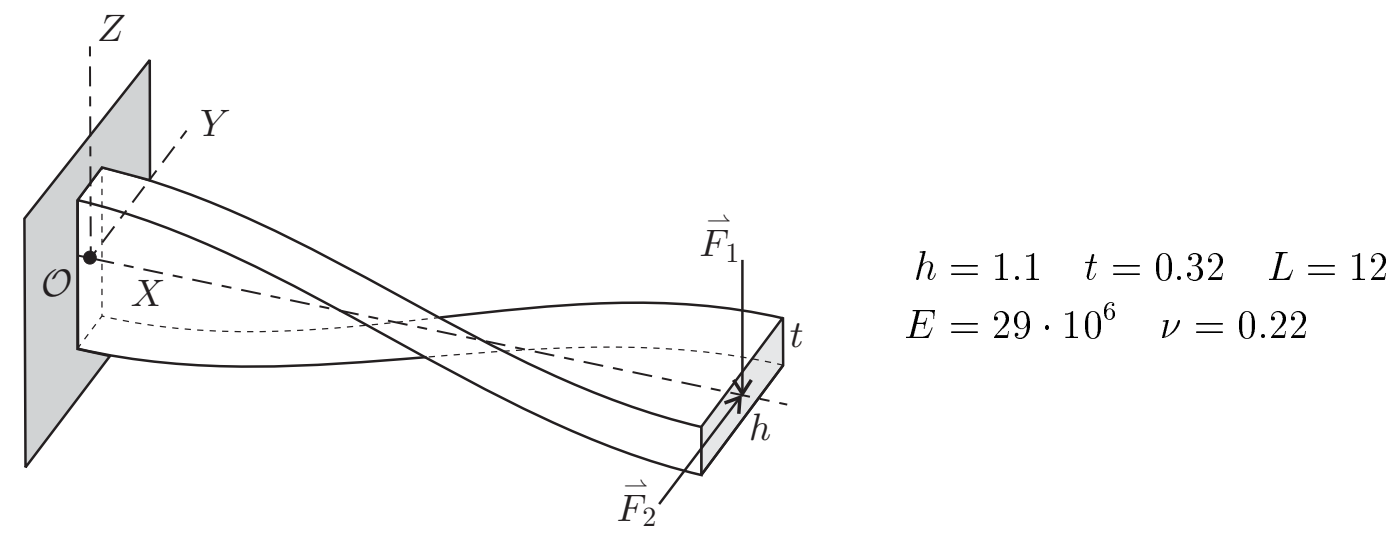

Fig. 14. Pretwisted beam for an angle of $\pi / 2$.

In Table 7 the results for the free-end displacements in the direction of the applied force are shown for each load case. The results of the present formulation are compared to the theoretical results presented in [22], to numerical solution of the beam theory of Dutta and White [13], and to the results of shell theory of Ibrahimbegovic and Frey [17].

It follows from Table 7 that the present formulation gives very accurate results even if only two low order elements are employed. The results of the present formulation converge to the values $u_{Z}=0.005429$ and $u_{Y}=0.001750$. The relative error is very small, especially in the first load case. In the second load case, the relative error is substantial only when a single linear element is employed. After refining the mesh or using quadratic elements, the relative error decreases to $0.23 \%$. The comparison with other studies shows the advantage of the present formulation. The results from [17] fully agree with the exact solution only for the second load case when the $12 \times 2$ mesh is used; if the finer mesh is used $(24 \times 4)$, the results differ again.

This test proves that the present formulation takes the initially non-planar configuration of the beam into account properly. It is also interesting to observe that both update procedures give the same results. This could be explained 
by the minor rate of rotational changes, compared to the initial rotations of the undeformed beam.

Table 7

Free-end displacements of a $\pi / 2$-pretwisted cantilever.

\begin{tabular}{crrrrr}
\hline & \multicolumn{3}{c}{ load case 1} & \multicolumn{3}{c}{ load case 2} \\
$E$ & $n_{e}$ & displacement $u_{Z}$ & error (\%) & displacement $u_{Y}$ & error (\%) \\
\hline$E_{2}$ & 1 & 0.005474 & 0.92 & 0.001517 & 13.51 \\
& 2 & 0.005429 & 0.09 & 0.001738 & 0.91 \\
& 3 & 0.005429 & 0.09 & 0.001748 & 0.34 \\
& 12 & 0.005429 & 0.09 & 0.001750 & 0.23 \\
$E_{3}$ & 1 & 0.005422 & 0.03 & 0.001750 & 0.23 \\
& 2 & 0.005429 & 0.09 & 0.001750 & 0.23 \\
\hline$[17]$ & $6 \times 1$ & 0.005390 & 0.63 & 0.001759 & 0.29 \\
& $12 \times 2$ & 0.005405 & 0.35 & 0.001754 & 0.00 \\
& $24 \times 4$ & 0.005411 & 0.24 & 0.001751 & 0.17 \\
\hline$[13]$ & 12 & 0.005402 & 0.41 & 0.001741 & 0.74 \\
\hline exact $[22]$ & & $\mathbf{0 . 0 0 5 4 2 4}$ & 0.00 & $\mathbf{0 . 0 0 1 7 5 4}$ & 0.00 \\
\hline
\end{tabular}

$E=$ type of element, $n_{e}=$ number of elements

\subsection{Cantilever, bent to a helical form}

We consider a very interesting example, first presented by Ibrahimbegovic [19]. When a straight in-plane cantilever is subjected to a point moment at its free end, it deforms into a part of a circle, which results in a pure bending of the cantilever. A much more interesting behaviour is observed when a small outof-plane point force is added at the free end of the cantilever (Figure 15a). The out-of-plane force causes the out-of-plane displacements of the beam. We took the same geometric and material properties of the cantilever as in [19]:

$$
\begin{aligned}
G A_{2} & =G A_{3}=E A_{1}=10^{4} \quad L=10 \\
E J_{2} & =E J_{3}=G J_{1}=10^{2} .
\end{aligned}
$$

The two loads $M=200 \pi \lambda$ and $F=50 \lambda$ increase incrementally from $\lambda=0$ to $\lambda=1$ in 1000 steps. The beam is modelled by a mesh of 25 elements 


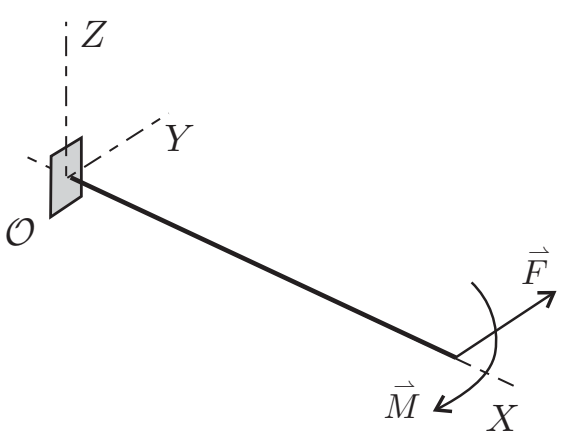

a)

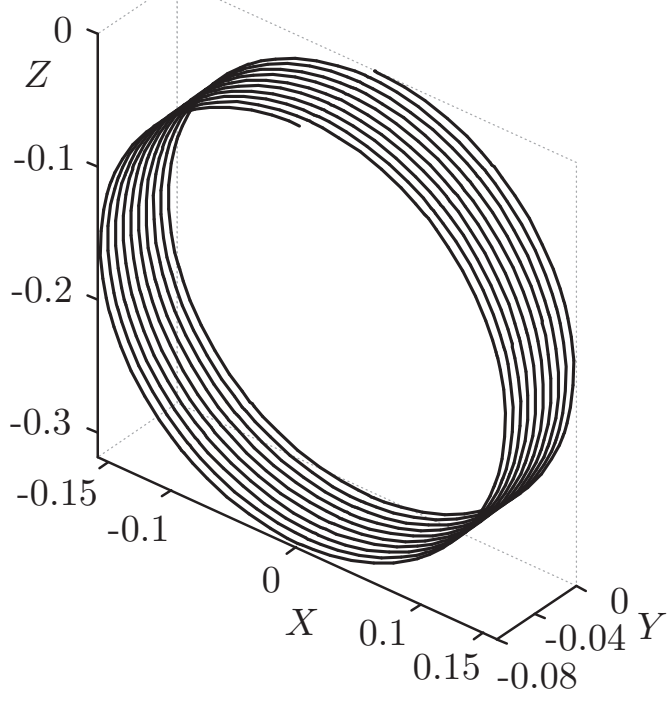

b)

Fig. 15. Cantilever, bent to the helical form: a) the undeformed beam and the loading, and b) the deformed beam.

with 8 interpolation points, and the more precise non-additive update is used. The result of a simultaneous application of moment and force is a beam, bent into a helical form (Figure 15b). For the values of geometrical and material properties as taken, the beam bends into ten circles, which do not lie in the same plane, yet very close together. The maximum out-of-plane displacement is roughly -0.08 . It must be pointed out that, at the final load stage, the beam is bent in the opposite direction to the applied force.

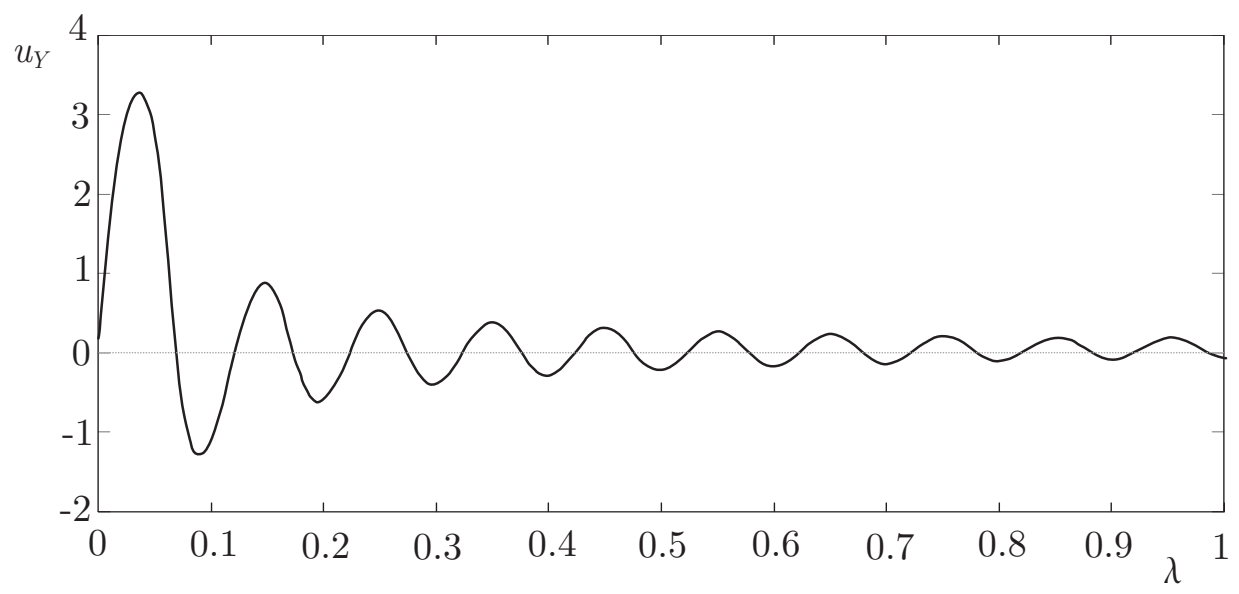

Fig. 16. Free end displacement, $u_{Y}$, versus loading factor, $\lambda$.

The displacement $u_{Y}$ of the free end of the cantilever as a function of loading factor $\lambda$ is shown in Figure 16. Observe that the curve, describing the displace- 
ment, oscillates around the zero value with its amplitude slowly decreasing. Thus, during the load increase, the beam bends to different sides of $X Z$-plane.

The analyses of Ibrahimbegovic [19] and later by Battini and Pacoste [5] show the importance of the suitable parametrization of rotations in order to obtain the correct results. The parametrization of rotations in the present formulation avoids problems of large rotations. The values of the free-end displacements almost completely agree with the results in [19] and [5].

\subsection{Cantilever $45^{\circ}$ bend}

This classical problem, presented by Bathe and Bolourchi [4], includes all modes of deformation of a structure: bending, shear, extension and torsion. Therefore, it represents a suitable test for the capability of the finite element. The initial axis of the cantilever is an arc of the circle of radius 100 located in the horizontal plane $(X, Y)$. The beam cross-section is a unit square. The load 600 is applied at the free end. Other material and geometric properties of the problem are shown in Figure 17.

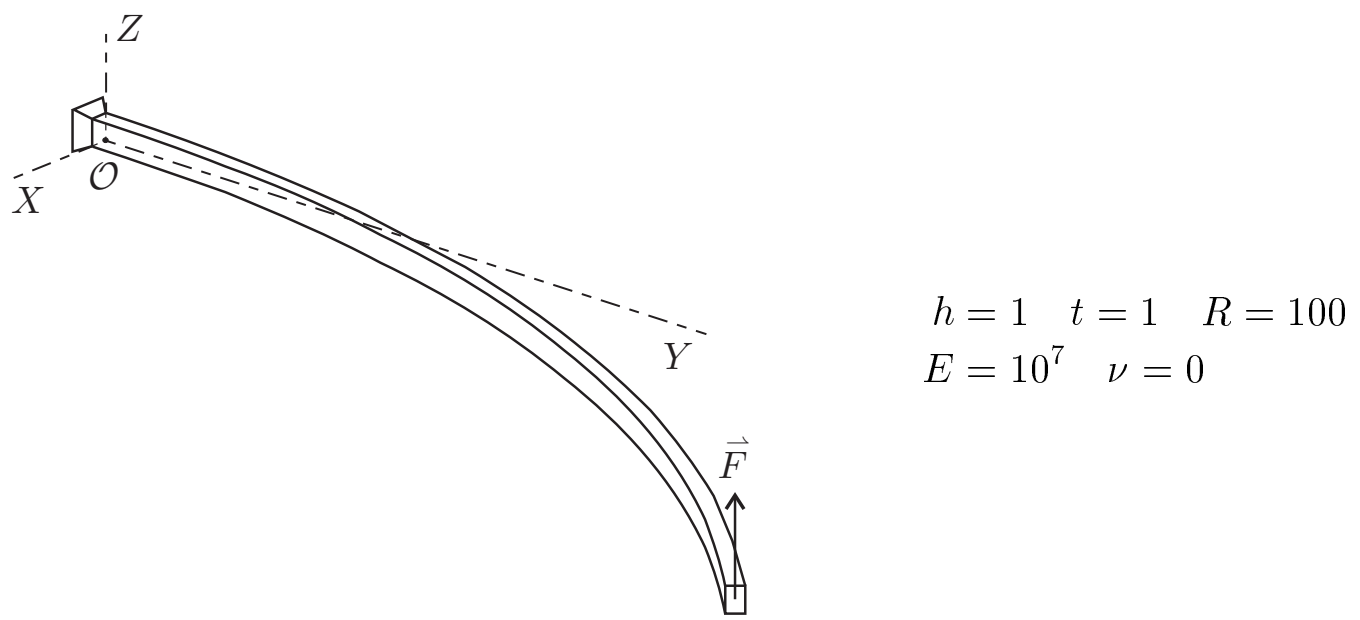

Fig. 17. Cantilever $45^{\circ}$ bend.

The numerical solution has been obtained by various researchers. When comparing the results we should be aware that they have reached the final loadstage in a different number of steps. We model the beam by 8 straight elements in all the cases and apply the non-additive update. Table 8 displays the comparison of the results of the present linear straight element $E_{2}$ to the results of other authors. The solution obtained by our element is in accord with other solutions. The difference between various solutions also arises from different simplifications used. We, for example, employed the torsional moment of inertia as suggested by the Coulomb theory of torsion, where $J_{1}=J_{2}+J_{3}$. 
No reliable result is available for this problem, so the results can be compared only qualitatively. Several authors have analyzed the rate of convergence of Newton's method. As far as the rate of convergence is concerned, the present formulation is privileged. The total number of iterations depends on the number of load steps used and the accuracy required. When a single load step is used, only 6 iterations are needed for accuracy tolerance $10^{-9}$. By increasing the number of load steps, the total number of iterations also increases, while the number of iterations in each particular load step remains roughly the same. It should be pointed out that the final results are not affected by the number of load steps which again confirms the path-independency of our formulation.

Table 8

Free-end position of the cantilever $45^{\circ}$ bend under out-of-plane force.

\begin{tabular}{rrrrrrrrr}
\hline & & & \multicolumn{3}{c}{$F=300$} & \multicolumn{4}{c}{$F=600$} \\
formulation & load steps & iterations & $r_{X}$ & $r_{Y}$ & $r_{Z}$ & $r_{X}$ & $r_{Y}$ & $r_{Z}$ \\
\hline$[4]$ & 60 equal & & 22.5 & 59.2 & 39.5 & 15.9 & 47.2 & 53.4 \\
{$[29]$} & $300,2 \times 150$ & $27=13+8+6$ & 22.33 & 58.84 & 40.08 & 15.79 & 47.23 & 53.37 \\
{$[6]$} & 6 equal & $7-8$ per increment & 22.14 & 58.64 & 40.35 & 15.55 & 47.04 & 53.50 \\
{$[10]$} & 6 equal & & 22.31 & 58.85 & 40.08 & 15.75 & 47.25 & 53.37 \\
{$[18]$} & 6 equal & 6 per increment & - & - & - & 15.68 & 46.96 & 53.41 \\
{$[27]$} & 2 equal & $11=6+5$ & - & - & - & 15.62 & 47.01 & 53.51 \\
\hline present $E_{2}$ & single & 6 & 22.28 & 58.78 & 40.16 & 15.74 & 47.15 & 53.43 \\
& 2 equal & $11=6+5$ & 22.28 & 58.78 & 40.16 & 15.74 & 47.15 & 53.43 \\
& 6 equal & $24=6^{*} 4$ & 22.28 & 58.78 & 40.16 & 15.74 & 47.15 & 53.43 \\
\hline
\end{tabular}

number of elements: 8 , type of element: linear, straight

Only slightly different results are obtained if the additive update is used (the use of 8 elements $E_{2}$ gives $\boldsymbol{r}=(15.64,47.21,53.46)$, see Table 8 for the comparison of the results).

\subsection{Illustration of the effects of inconsistency of equilibrium at cross-sections}

The discussion on the reasons and consequences of the inconsistency of equilibrium at cross-sections in standard finite-element formulations is beyond the scope of this article. We wish only to demonstrate the importance of the consistency being satisfied by a single numerical example. As the effects of the inconsistency are much more pronounced for non-linear material we also study a materially non-linear concrete beam. We compare the numerical results for internal forces using two formulations: (i) the present consistent formulation; and (ii) a slightly changed formulation in which the consistency conditions 
(104)-(105) are satisfied only in their weak (variational) form. This means that the tangent stiffness matrix remains the same, but the values of the first $6 N$ components of the residual vector are set to zero.

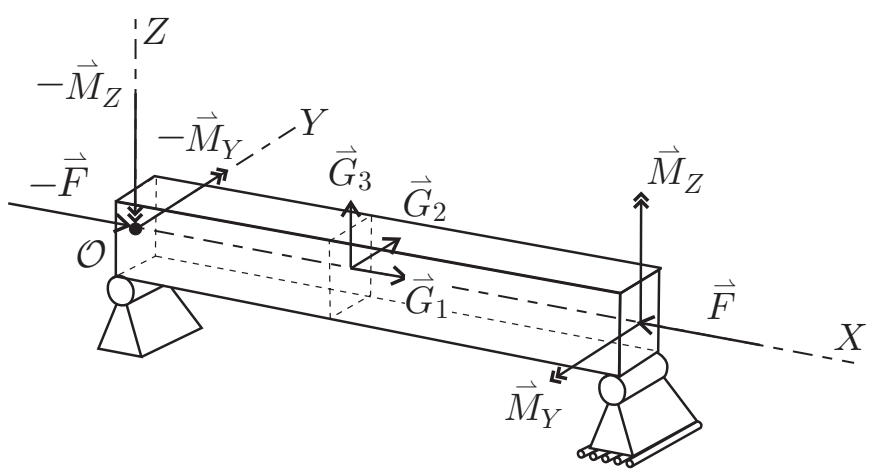

Fig. 18. Simply supported beam subjected to axial forces and bending moments at ends.

We consider a simply supported beam subjected to the opposite axial forces and bending moments at its both ends (see Figure 18). The beam length is $L=120$ and its cross-section is rectangular with size $10 \times 20$. Our results are shown for the axial force $F=100$ and bending moments $M_{Y}=200 \sqrt{3}$ and $M_{Z}=200$. We used 10 and 50 elements $E_{8}$ while enforcing the weak form of the consistency condition. Two different types of material of the beam are considered: linear elastic material and concrete. Both, the constitutive force and moment vectors as well as the equilibrium force and moment, are evaluated and compared with respect to the material basis.

We first analyze the linear elastic beam with elastic modulus $E=3000$ and shear modulus $G=1500$. Our results for the stress resultants are shown in Figure 19. From Figure 19 we observe that the inconsistency does have some effect in the linear-elastic-material problems; however, the discrepancies are small. The differences for bending moments become even smaller with an increase of the number of elements, while for the constitutive forces and torsional moment, the increase of the number of elements does not affect the results. Note that if we the use of the present consistent formulation, the curves, representing the constitutive and equilibrium stress-resultants, completely agree. 

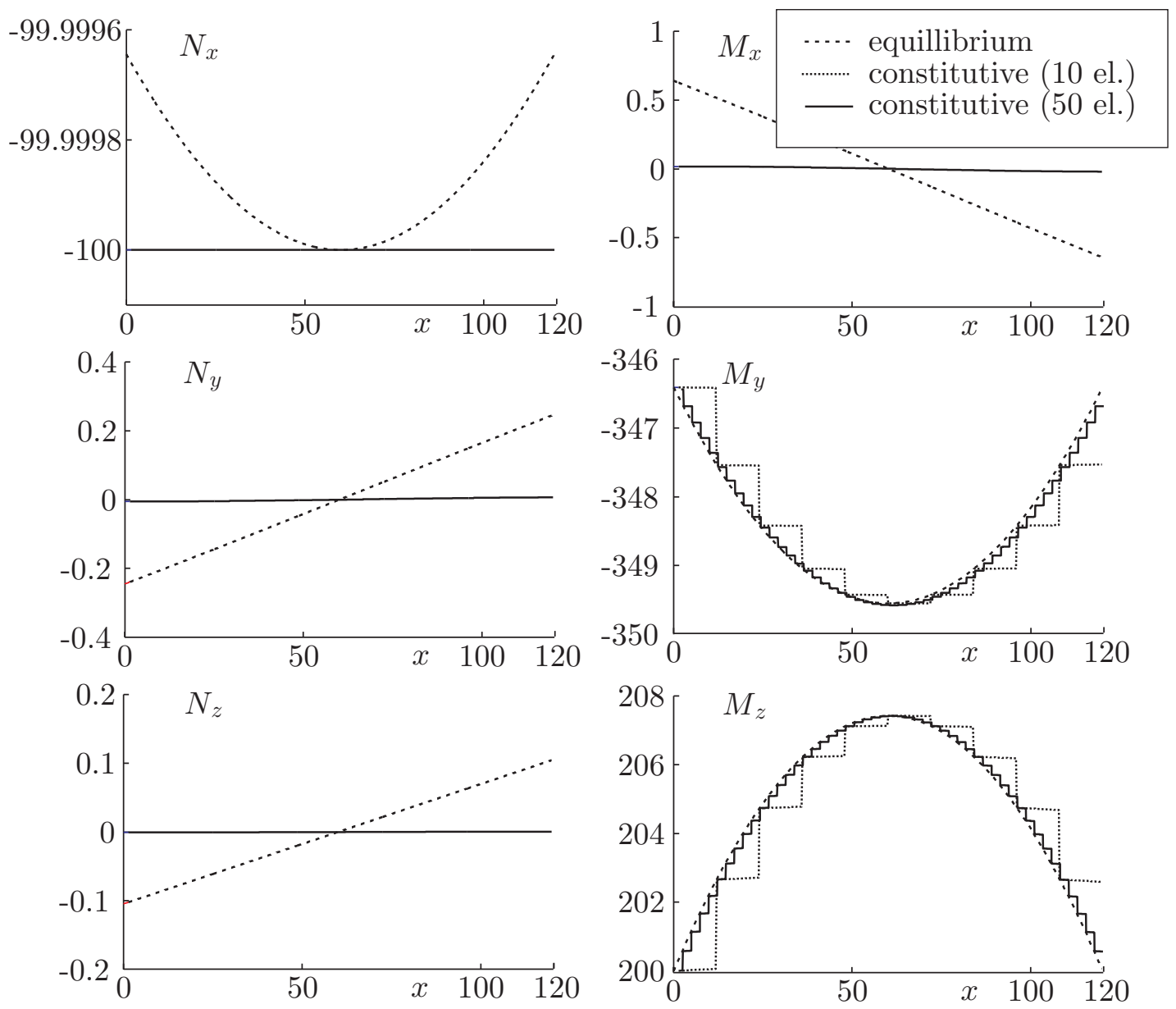

Fig. 19. Inconsistency of equilibrium at cross-section for linear material.

Much greater differences are found if a concrete material is used. We show the results for the concrete beam using the stress-strain law proposed by Desayi and Krishnan [12] with strength of concrete in compression $f_{\mathrm{m}}=3$, the strain at peak stress $\varepsilon_{1}=-0.002$, and the ultimate strain at compression $\varepsilon_{\mathrm{u}}=-0.008$. The bearing capacity of concrete in tension is ignored. The calculated stress-resultants variations are shown in Figure 20. In contrast to the previous case, we now observe considerable differences in bending moments and axial force. It is interesting that graphs of the equilibrium and constitutive bending moments have similar shapes, but have very different amplitudes. By the increase of the number of elements the shapes of the curves representing constitutive bending moments approach those of the equilibrium moments, but the curves remain equally apart. Note that by the use of the present consistent formulation, the curves, representing the constitutive and equilibrium stress-resultants, again completely agree. 

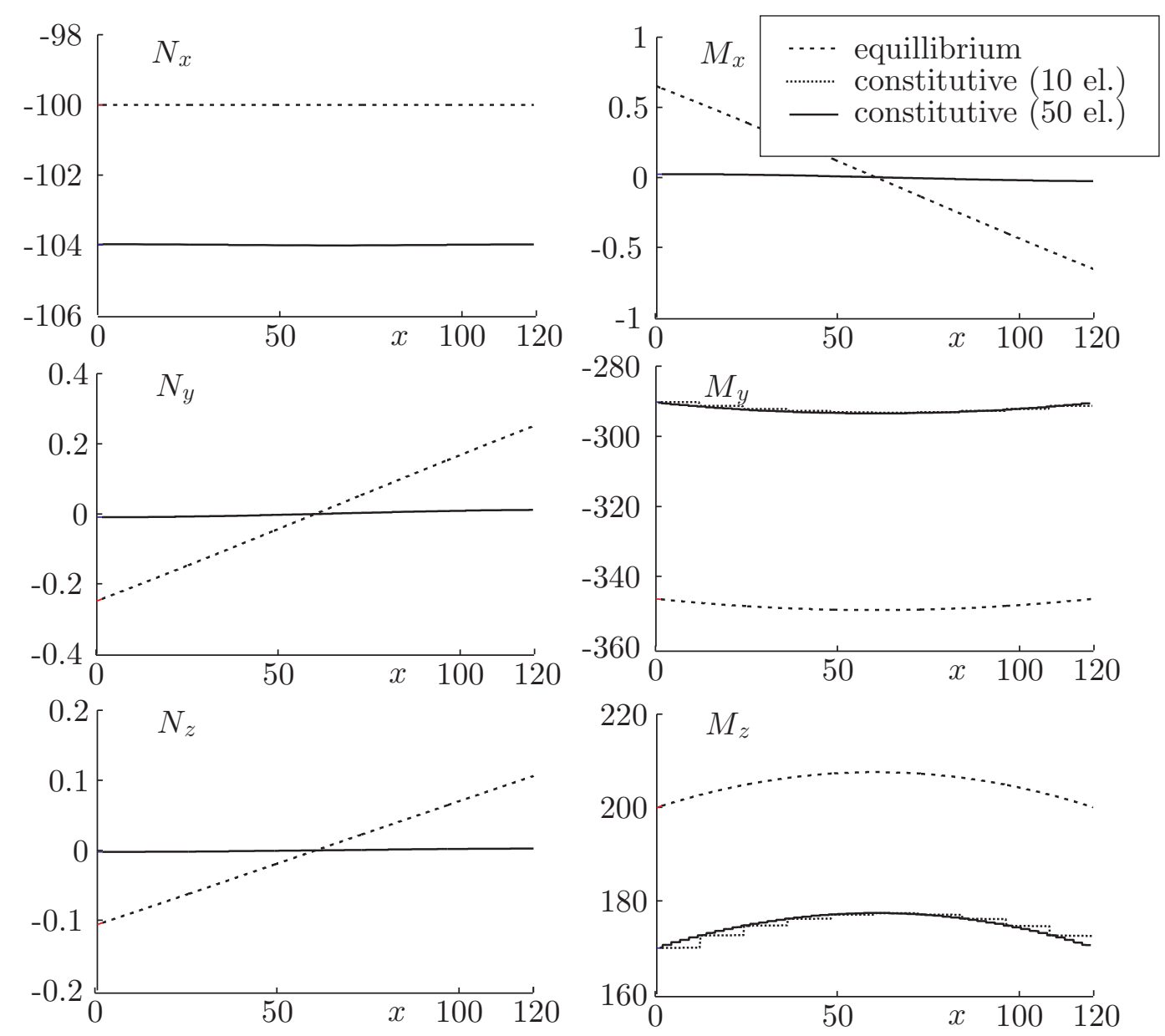

Fig. 20. Inconsistency of equilibrium at cross-section for non-linear material.

\section{Conclusions}

We presented a new finite element formulation of the geometrically exact threedimensional beam theory which is based on the interpolation of strain vectors. The essential points of the formulation are as follows:

(i) A modified principle of virtual work is proposed in which the strain vectors are the only unknown functions. Hence it follows that the strain vectors are the only functions that need to be interpolated. The formulation based on the strain interpolation is inherently strain-objective, path-independent and has many advantages in materially non-linear problems, such as in localization of strains in concrete structures due to the softening of material.

(ii) Displacement and rotational vectors (or their variations) are not interpolated.

(iii) The interpolation of both strain vectors increases the total number of internal degrees of freedom. These are condensed on an element level, so that the number of external degrees of freedom still remains 12. 
(iv) The consistency condition that the equilibrium and the constitutive internal force and moment vectors are equal, is enforced to be satisfied at the given set of points (the 'collocation'). This considerably improves the accuracy of the internal forces and moments at these points, which is of utmost importance particularly in materially non-linear problems and composite structures where the accuracy of interlayer forces is essential for the overall results.

(v) The determination of internal forces and moments does not require the differentiation. It then follows that the accuracy of the internal forces and moments is of the same order as the accuracy of the basic variables. This is an important advantage compared to formulations where the derivatives are needed for the evaluation of internal forces.

(vi) The matrices of a finite element are, from the outset on, derived with respect to the global coordinate system. The coordinate transformation from the local to the global system is thus avoided. An arbitrary initial bending and/or twisting curvature and deformation of the beam can be prescribed at the unloaded configuration, so that the beam can have an arbitrary bending, shear, and torsional deformed initial shape.

(vii) The present finite elements are free of locking.

(viii) A number of finite elements of different order have been tested by various numerical examples. A rapid convergence and an outstanding accuracy are characteristic for the higher-order elements.

\section{Acknowledgment}

This work was supported by the Ministry of Education, Science and Sport of the Republic of Slovenia through the grant S2-792-016/19121. The support is gratefully acknowledged.

\section{References}

[1] J. H. Argyris, "An excursion into large rotations", Comput. Methods Appl. Mech. Eng. 32, 85-155, 1982.

[2] J. H. Argyris, P. C. Dunne, G. Malejannakis, D. W. Scharpf, "On large displacements - small strain analysis of structures with rotational degrees of freedom", Comput. Methods Appl. Mech. Eng. 14, 99-135, 1978.

[3] S. N. Atluri, A. Cazzani, "Rotations in computational solid mechanics", Arch. Comput. Methods in Eng. 2, 49-138, 1995.

[4] K. J. Bathe, S. Bolourchi, "Large displacement analysis of three-dimensional beam structures", Int. J. Numer. Meth. Eng. 14, 961-986, 1979. 
[5] J. Battini, C. Pacoste, "Co-rotational beam elements with warping effects in instability problems", Comp. Methods Appl. Mech. Eng. 191, 1755-1789, 2002.

[6] A. Cardona, M. Géradin, "A beam finite element non-linear theory with finite rotations", Int. J. Numer. Meth. Eng. 26, 2403-2438, 1988.

[7] J. Choi, J. Lim, "General curved beam elements based on the assumed strain fields", Comput. Struct. 55, 379-386, 1995.

[8] M. A. Crisfield, "A consistent co-rotational formulation for non-linear, threedimensional beam elements", Comp. Meth. Appl. Mech. Engng. 81, 131-150, 1990 .

[9] M. A. Crisfield, G. Jelenić, "Objectivity of strain measures in the geometrically exact three-dimensional beam theory and its finite-element implementation", Proc. Roy. Soc. London A 455, 1125-1147, 1999.

[10] L. A. Crivelli, C. A. Felippa, "The three-dimensional non-linear Timoshenko beam based on the core-congruential formulation", Int. J. Numer. Meth. Eng. 36, 3647-3673, 1993.

[11] D. A. DaDeppo, R. Schmidt, "Instability of clamped-hinged circular arches subjected to point load", ASME J. Appl. Mech. 97, 894-896, 1975.

[12] P. Desayi, S. Krishnan, "Equation for the stress-strain curve of concrete", Journal of American Concrete Institute 61, 345-350, 1964.

[13] A. Dutta, D. W. White, "Large displacement formulation of three-dimensional beam element with cross-sectional warping", Comput. Struct. 45, 9-24, 1992.

[14] Z. Friedman, J. B. Kosmatka, "An accurate two-node finite element for shear deformable curved beams", Int. J. Numer. Meth. Eng. 41, 473-498, 1998.

[15] M. Géradin, D. Rixen, "Parametrization of finite rotations in computational dynamics: a review", Revue européenne des éléments finis 4, 497-553, 1995.

[16] N. Hu, B. Hu, B. Yan, H. Fukunaga, H. Sekine, "Two kinds of $C^{0}$-typed elements for buckling analysis of thin-walled curved beams", Comput. Meth. Appl. Mech. Eng. 171, 87-108, 1999.

[17] A. Ibrahimbegovic, F. Frey, "Stress resultant geometrically non-linear shell theory with drilling rotations. Part III: linearized kinematics", Int. J. Numer. Methods Eng. 37, 3659-3683 1994.

[18] A. Ibrahimbegovic, "On the finite element implementation of geometrically nonlinear Reissner's beam theory: 3d curved beam element", Comput. Methods Appl. Mech. Eng. 122, 11-26, 1995.

[19] A. Ibrahimbegovic, "On the choice of finite rotation parameters", Comput. Methods Appl. Mech. Eng. 149, 49-71, 1997.

[20] A. Ibrahimbegovic, R. L. Taylor, "On the role of frame-insurance in structural mechanics models at finite rotations", Comput. Methods Appl. Mech. Eng., 191, 5159-5176, 2002. 
[21] G. Jelenić, M. Saje, "A kinematically exact space finite strain beam model-finite element formulation by generalized virtual work principle", Comput. Methods Appl. Mech. Eng. 120, 131-161, 1995.

[22] R. H. MacNeal, R. L. Harder, "A proposed standard set of problems to test finite element accuracy", Finite Element. Anal. Design 1, 3-20, 1985.

[23] B. Nour-Omid, C. C. Rankin, "Finite rotation analysis and consistent linearization using projectors", Comput. Methods Appl. Mech. Eng. 93, 353384, 1991.

[24] I. Planinc, M. Saje, B. Čas, "On the local stability condition in the planar beam finite element", Structural Engineering and Mechanics 12(5), 507-526, 2001.

[25] E. Reissner, "On finite deformation of space-curved beams", J. Appl. Math. Phys. 32, 734-744, 1981.

[26] M. Saje, G. Turk, A. Kalagasidu, B. Vratanar, "A kinematically exact finite element formulation of elastic-plastic curved beams", Comput. Struct. 67, 197214, 1998.

[27] M. Schulz, F. C. Filippou, "Non-linear spatial Timoshenko beam element with curvature interpolation”, Int. J. Numer. Methods Eng. 50, 761-785, 2001.

[28] J. C. Simo, "A finite strain beam formulation. The three-dimensional dynamic problem. Part I", Comput. Methods Appl. Mech. Eng. 49, 55-70, 1985.

[29] J. C. Simo, L. Vu-Quoc, "A three-dimensional finite-strain rod model. Part II: Computational aspects”, Comput. Methods Appl. Mech. Eng. 58, 79-116, 1986.

[30] W. M. Smoleński, "Statically and kinematically exact nonlinear theory of rods and its numerical verification", Comput. Methods Appl. Mech. Eng. 178, 89113,1999 .

[31] R. A. Spurrier, "Comment on Singularity-free extraction of a quaternion from a direction-cosine matrix", J. Spacecraft 15, 255, 1978.

[32] B. Tabarrok, M. Farshad, H. Yi, "Finite element formulation of spatially curved and twisted rods", Comput. Methods Appl. Mech. Eng. 70, 275-299, 1988.

[33] B. Vratanar, M. Saje, "A consistent equilibrium in a cross-section of an elasticplastic beam", Int. J. Solids Structures 36, 311-337, 1999.

[34] S. P. Timoshenko, J. M. Gere, "Theory of Elastic Stability”, McGraw-Hill, New York, 1961. 